\title{
Rursus
}

Russus

Poiétique, réception et réécriture des textes antiques

$10 \mid 2017$

Traductions latines de textes grecs

\section{La traduction dans le domaine hagiographique médiolatin : l'apport de la linguistique quantitative}

Translation and Latin Hagiography : the contribution of quantitative linguistics

\section{Caroline Philippart}

\section{OpenEdition}

Journals

Édition électronique

URL : http://journals.openedition.org/rursus/1285

DOI : $10.4000 /$ rursus. 1285

ISSN : 1951-669X

Éditeur

Université Nice-Sophia Antipolis

Référence électronique

Caroline Philippart, « La traduction dans le domaine hagiographique médiolatin : l'apport de la

linguistique quantitative », Rursus [En ligne], 10 | 2017, mis en ligne le 30 août 2017, consulté le 19 avril 2019. URL : http://journals.openedition.org/rursus/1285; DOI : 10.4000/rursus. 1285

Ce document a été généré automatiquement le 19 avril 2019

Rursus 


\title{
La traduction dans le domaine hagiographique médiolatin : l'apport de la linguistique quantitative
}

Translation and Latin Hagiography : the contribution of quantitative linguistics

\author{
Caroline Philippart
}

\section{La traduction dans le domaine hagiographique médiolatin}

\section{Un mode de diffusion courant, mais peu étudié}

1 La production hagiographique est l'une des plus riches du Moyen Âge ${ }^{1}$. Au fil des siècles, le corpus latin des vitae sanctorum et des passiones martyrum s'est enrichi par l'introduction de nouveaux textes, par la réécriture et l'adaptation d'anciens remis au goût du jour, ainsi que par la traduction de textes issus de l'étranger. Si le phénomène de la réécriture hagiographique en Occident latin est bien connu et a récemment fait l'objet d'une excellente synthèse par Monique GOULLET (2005), la pratique de la traduction dans ce domaine, en revanche, reste peu étudiée. Nombreux pourtant sont les textes hagiographiques issus du monde byzantin et entrés dans le monde latin par le biais d'une traduction. Une des plus anciennes et des plus célèbres de ces traductions est celle de la Vie d'Antoine par Athanase, traduite d'abord par un anonyme, à une date inconnue, puis, en 357, par Évagre d'Antioche, ami de saint Jérôme. La plupart des Passions grecques des martyrs orientaux, des Vies des Pères et des saintes femmes pénitentes sont traduites en latin dès le $\mathrm{VI}^{\mathrm{e}}$ siècle. L'activité de traduction se poursuit, avec plus ou moins de succès, et s'amplifie au IX siècle, à Rome sous la plume d'Anastase le Bibliothécaire, à Naples grâce au travail d'un cercle de traducteurs $\left(\mathrm{IX}^{\mathrm{e}}-\mathrm{X}^{\mathrm{e}}\right)$, puis à Amalfi au XI $\mathrm{XI}^{\mathrm{e}}$ siècle. Malgré l'abondance de la production, la traduction dans le domaine hagiographique médiolatin 
n'a jamais suscité auprès des chercheurs modernes autant d'intérêt que la traduction qui s'est pratiquée durant l'Antiquité classique et tardive ou que celle qui s'est développée à la Renaissance.

\section{Obstacles à l'étude de la traduction hagiographique}

Pour dresser un panorama des traductions dans le domaine hagiographique médiolatin, il manque une histoire générale des traductions médiolatines. Il existe quelques répertoires sur lesquels s'appuyer, comme l'ouvrage de A. SIEGMUND, Die Überlieferung der griechischen christlichen Literatur in der lateinischen Kirche bis zum zwölften Jahrhundert, mais aussi précieux soit-il, celui-ci date de 1947 et devrait être actualisé en fonction des résultats récents de la recherche : de nouveaux textes identifiés comme des traductions devraient être ajoutés à la liste, d'autres dont l'origine latine a été démontrée devraient en être retirés ${ }^{2}$. Le livre de W. BERSCHIN, Griechisch lateinisches Mittelalter : von Hieronymus zu Nikolaus von Kues, paru en 1980, donne un panorama très riche sur les contacts entre le grec et le latin durant le Moyen Âge. Malheureusement, le corpus des traductions hagiographiques médiolatines reste loin d'être établi de manière définitive. De nombreux textes n'ont pas encore été édités, le dépouillement des manuscrits médiévaux est loin d'être achevé, et, parmi les textes édités, beaucoup n'ont pas encore été étudiés.

3 L'étude des traductions hagiographiques souffre donc d'un manque cruel d'éditions et d'études critiques, confrontant traduction latine et modèle grec (CHIESA, 1995 : 169-170, 1987a : 1). La principale difficulté réside dans le caractère mobile de la tradition littéraire, qui complique sérieusement l'identification des traductions et l'établissement du texte. En effet, une fois traduits, les textes ont été employés de la même manière que ceux qui ont été rédigés directement en latin. Ils ont connu les mêmes modes de circulation et de diffusion et ont fait l'objet de retouches et de remaniements au même titre que les autres. Il n'est, par conséquent, pas toujours aisé d'identifier avec certitude les traductions, de distinguer le texte traduit du texte non traduit, ou encore de retrouver le texte grec qui a servi de modèle à la version latine. Certaines variantes peuvent faire penser que le traducteur a utilisé une version légèrement différente du texte grec que l'on a conservé. Dans ce cas, on peut se demander si le texte latin n'est pas la traduction d'un remaniement du texte grec conservé ou si le texte grec conservé n'est pas lui-même un remaniement du texte qui a servi de modèle à la traduction latine. À moins que la version latine ne soit le remaniement d'une première traduction du texte grec. Parmi les textes publiés, un petit nombre seulement a fait l'objet d'une étude aussi approfondie que la Pénitence de Pélagie (PETITMEnGin, 1981) ou la Passion de Fébronie (CHIESA, 1990).

\section{Contexte historique : quelques repères}

4 Pour appréhender les traductions dans le domaine hagiographique médiolatin, il faut les replacer dans leur contexte, à savoir celui du développement de la pratique depuis l'Antiquité et les première traductions chrétiennes, avec les différents modèles et procédés possibles, celui des circonstances et des motifs qui ont donné lieu aux traductions, celui de la connaissance du grec en Occident et des compétences des traducteurs hagiographes.

5 Malheureusement, la plupart des traductions latines des Vies et des Passions grecques sont non signées et dépourvues de prologue. Il est donc très difficile de les dater et de les 
localiser, et quasiment impossible de les attribuer. Seul un petit nombre de traducteurs nous est connu, en particulier à partir du IX siècle quand les traductions se dotent d'un prologue $^{3}$. Prologues, épilogues, préfaces, postfaces et dédicaces font partie du paratexte, c'est-à-dire de tout ce qui ne fait pas à proprement parler partie du texte, mais qui l'entoure et le prolonge pour le présenter (GENETTE, 1987: 7-8). Dans les prologues, les hagiographes s'identifient souvent, évoquent leur commanditaire ou leur dédicataire, énoncent le motif de la traduction (parce qu'il n'en existe pas encore ou pour remplacer une traduction mauvaise ou de faible qualité littéraire), ils indiquent parfois leur source d'information, critiquent à l'occasion leur modèle, présentent la méthode suivie; ils ont aussi recours à la captatio benevolentiae, en proclamant leur souci de véracité et leur incompétence stylistique et en faisant appel à l'indulgence des lecteurs. Les prologues sont donc une source importante pour la connaissance des traductions hagiographiques, même s'il ne faut pas se laisser abuser par les topoi dont ils sont truffés. À côté des topoi rhétoriques classiques et de ceux qui ressortissent à la littérature chrétienne, il y a ceux qui sont spécifiques aux traductions. Ainsi, quand les hagiographes ajoutent un prologue à leurs traductions, ils ont l'habitude de citer le nom des grands traducteurs chrétiens, en particulier celui de Jérôme, et de se positionner par rapport à leur théorie, en choisissant entre le mode littéral ad verbum et le mode plus libre mais plus fidèle au sens ad sensum ( CHIESA, 1987a).

6 Le but des traductions hagiographiques était de diffuser les Vies des saints et les Passions des martyrs grecs et orientaux peu connus en Occident ou difficilement accessibles et de les propager dans un large public, en particulier pour les besoins de la pastorale. Il fallait donc que les œuvres traduites soient à la fois abordables et plaisantes. Dans son article Ad verbum o ad sensum, Paolo CHIESA (1987a) présente les différents modèles de traduction et pose la question de la conscience méthodologique chez les traducteurs de l'Antiquité tardive et du haut Moyen Âge. Ailleurs (1989, 1989-1990), il s'intéresse aux techniques de traduction et aux traducteurs hagiographes des $\mathrm{IX}^{\mathrm{e}}$ et $\mathrm{X}^{\mathrm{e}}$ siècles. Il montre ainsi, qu'en l'absence de prologue ou d'éléments explicites, le mode de traduction et certaines caractéristiques peuvent aider à dater et à localiser les textes.

7 Les traducteurs du Moyen Âge et a fortiori les traducteurs hagiographes ne pouvaient ignorer la tradition chrétienne en matière de traduction. Influencés par les commentaires de Saint Jérôme et de Rufin, ils pouvaient choisir d'y adhérer ou de s'y opposer, en l'exprimant ou non dans un prologue. Le mode utilisé n'était toutefois pas toujours le résultat d'un choix conscient de la part du traducteur, il pouvait dépendre de plusieurs facteurs à la fois : époque, lieu, niveau culturel, compétences linguistiques, finalité de la traduction, public visé, etc.

8 Après la grande floraison du IV siècle, l'activité de traduction se poursuit aux $\mathrm{V}^{\mathrm{e}}$ et $\mathrm{VI}^{\mathrm{e}}$ siècles, en raison de l'ignorance de plus en plus grande du grec (BARDY, 1948 : 155-229). Les traductions sont principalement anonymes, même si on sait qu'à Rome un moine scythe du nom de Denys le Petit a traduit plusieurs Vies grecques. Dans la seconde moitié du VIe siècle, la production connaît un déclin, qui correspond à une baisse générale du niveau intellectuel occidental en ces temps troublés. Dans le domaine hagiographique, il est possible qu'il y ait eu des traductions dans le Nord (CHIESA, $1990: 111-118$ ) et plus que vraisemblable qu'il y en ait eu en Campanie ; mais la seule attestation d'une activité de traduction stable et organisée se trouve à Rome, qui est restée un lieu privilégié pour les relations entre l'Occident et l'Orient et qui verra d'ailleurs l'installation de plusieurs monastères grecs dès le VII ${ }^{e}$ siècle (SANSTERRE, 1983: I, 73 et 150). C'est de Rome 
précisément que serait issue la majorité des traductions hagiographiques, entre la seconde moitié du $\mathrm{VI}^{\mathrm{e}}$ siècle et la première moitié du IX siècle (SIEGMUND, 1947 : 195-277). Ces traductions, généralement anonymes, sont caractérisées par un très grand littéralisme, avec une correspondance quasi parfaite entre le grec et le latin. Fidèles aux structures de l'original, notamment dans l'ordre des mots, elles sont souvent maladroites, parfois incompréhensibles, ce qui leur valut d'être taxées de rustico stylo.

9 À partir du IX siècle, les traductions se dotent plus fréquemment d'un prologue ou d'un épilogue. Les traducteurs sortent de l'anonymat et présentent leurs versions. De manière générale, leurs traductions sont plus libres et plus respectueuses de la langue latine, elles présentent aussi une plus grande qualité littéraire: les traducteurs cherchent des équivalents latins pour les structures grecques, afin de rendre le texte intelligible et de lui assurer une meilleure diffusion. Les traducteurs apparaissent plus cultivés et semblent avoir une meilleure connaissance du grec (CHIESA, 2002 : 184-185). À Rome, Anastase le Bibliothécaire est l'auteur de nombreuses traductions (LEONARDI, 1981). Si celles-ci gardent un côté littéral, elles sont loin du littéralisme rigoureux qui caractérise les traductions des siècles précédents. Anastase se montre à la fois respectueux de l'œuvre originale et soucieux de fournir une version intelligible et correcte en latin; on parle chez lui de littéralisme intelligent (CHIESA, 1987b: 882). Les prologues qu'il ajoute à ses traductions témoignent d'ailleurs de sa réflexion méthodologique et de son implication en tant que traducteur.

10 La région de Naples est un autre foyer de traductions hagiographiques aux $\mathrm{IX}^{\mathrm{e}}$ et $\mathrm{X}^{\mathrm{e}}$ siècles. On y rattache un nombre important de traductions, ainsi que le nom de neuf traducteurs: l'évêque Athanase II, le prêtre Ursus, les diacres Paul et Jean, les sous-diacres Bonitus et Pierre, les clercs Cicinnio et Grégoire, et Guarimpotus (D'ANGELO, $2007: 68-84)^{4}$. Le duché napolitain est encore étroitement lié à Byzance sur le plan politique, des minorités hellénophones y sont installées et les échanges entre les deux cultures sont importants (LuZzATI LAGANÀ, 1982). Vers la fin du IX siècle, l'épiscopat de Naples aurait lancé une vaste entreprise de révision de la tradition hagiographique locale, dans le but de la rendre plus adaptée au culte et à la prédication (GRANIER, 1995 et 2002). On parle d'école pour décrire ce groupe de traducteurs hagiographes, tant ils présentent de similitudes: outre le projet commun de doter l'Église d'un corpus de textes hagiographiques de bonne qualité, leurs œuvres montrent une relative homogénéité dans le style, dans la technique et dans l'emploi de références et de topoi. Ces traducteurs sont généralement présentés comme ayant une conception très libre de la traduction. Cependant, François DOLBEAU (1989) a souligné l'ambiguïté des termes traducteur et traduction pour désigner cette école, dont la production va de la rédaction de textes originaux en latin à la révision d'anciennes versions traduites de manière médiocre ou incorrecte, et à la rédaction de nouvelles traductions de textes pour lesquels on ne connaissait pas de traduction antérieure (aussi CHIESA, 1989-1990: 68). La confusion vient $\mathrm{du}$ fait que les hagiographes ne font pas de distinction claire entre ces différentes formes d'écrits. Dans les prologues, ils présentent leurs œuvres comme celles de traducteurs, qu'il s'agisse de véritables traductions ou de remaniements, au moyen d'une topique multiséculaire et d'une rhétorique empruntée à Jérôme, Rufin ou Évagre. François Dolbeau a, en outre, mis en évidence le rôle des interprètes dans le processus de rédaction : beaucoup de ces hagiographes ne comprenaient pas, ou mal, le grec, et étaient forcés de faire appel à des interprètes pour en donner une première traduction littérale, qu'ils retravaillaient ensuite pour l'améliorer sur les plans linguistique et stylistique. Il 
convient donc de répartir en deux catégories les prétendues traductions de l'école napolitaine, avec d'une part des adaptations assez libres de versions commandées à des interprètes grecs, qui étaient alors les seuls à avoir eu un contact direct avec le grec, et, d'autre part, des réécritures en beau style de traductions tombées en discrédit, parce que trop littérales.

11 Après le $\mathrm{X}^{\mathrm{e}}$ siècle, l'activité napolitaine se ralentit. La pratique de la traduction hagiographique semble s'être déplacée vers Amalfi, qui maintient des contacts avec Constantinople et reste un lieu d'échanges privilégié entre la culture grecque d'Orient et la culture latine d'Occident. Vers l'an mille, la petite ville d'Amalfi dispose d'un comptoir à Constantinople et d'un monastère au mont Athos. Les traducteurs amalfitains y furent particulièrement actifs au XI ${ }^{\mathrm{e}}$ siècle (BERSCHIN, 1989).

\section{Présentation de la méthode et du corpus}

\section{Objectif et méthodologie}

L'objectif de ma thèse (PHILIPPART DE FOY, 2008) était d'aborder la question de la traduction dans le domaine hagiographique médiolatin d'une manière originale, en étudiant le latin utilisé par les traducteurs pour rendre leur modèle grec et les techniques qu'ils ont adoptées pour le traduire, en considérant les traductions non pas une à une, mais d'une manière comparative au moyen de l'analyse quantitative. Bien consciente des lacunes embarrassantes qui entourent le sujet et reconnaissant la nécessité et la priorité du travail éditorial, je me suis demandé si, face à l'ampleur de la tâche qui restait à accomplir, on ne pouvait pas déjà mettre à profit ce qui était à disposition. Suffisamment de textes étaient édités pour permettre des comparaisons, même si des réserves devaient être émises sur la qualité de certaines éditions et si dès lors une certaine prudence était de mise dans l'interprétation des résultats. Il ne s'agissait pas pour moi d'écrire une étude particulière sur une traduction et son modèle ou sur un traducteur et ses traductions, mais bien d'aborder la traduction hagiographique d'une manière plus générale, en me basant sur l'analyse d'un corpus représentatif, formé à partir de textes édités, et en l'étudiant au moyen des méthodes quantitatives afin de caractériser et de classer les traductions sur la base de critères linguistiques, d'identifier et de qualifier des pratiques, des traditions localisées, des spécificités d'école ou de traducteur. L'idée était de procéder à différentes analyses en comparant le corpus, d'abord pris dans son ensemble, à un corpus de textes non traduits, donc de manière externe, puis de procéder à des comparaisons internes entre différents sous-corpus de traductions. Le premier type de comparaison avait pour objectif de mettre en évidence les caractéristiques des traductions en général, le second de dégager les spécificités de chaque groupe de traductions.

La statistique a été choisie comme méthode d'expertise, car elle présente l'avantage de pouvoir traiter un corpus important, de comparer tous les textes entre eux, en mettant en évidence les phénomènes linguistiques, morphologiques, syntaxiques ou lexicologiques, qui les caractérisent, les rapprochent ou les opposent (ÉVRARD, MELLET, 1998). Elle peut ainsi mener à une classification des textes selon les paramètres envisagés. $\mathrm{Vu}$ que les méthodes quantitatives reposent, faut-il le rappeler, sur des données chiffrées, les textes ont dû être traités de manière à permettre le décompte des différents éléments qui les constituent et qui sont susceptibles de les caractériser. La technique d'étiquetage 
du LASLA (Laboratoire d'Analyse Statistique des Langues Anciennes) de l'Université de Liège est apparue d'emblée la plus intéressante, car elle accompagne la lemmatisation d'une analyse morphosyntaxique, qui augmente considérablement les possibilités de recherche ${ }^{5}$. Dans cette étude sur la langue des traducteurs, la syntaxe est un aspect tout aussi important à envisager que le lexique. Les catégories grammaticales et énonciatives présentent en outre l'avantage d'être moins soumises à l'influence du thème que le vocabulaire. Elles peuvent elles aussi révéler des spécificités d'écriture et, dans ce cas, des procédés de traduction.

\section{Corpus}

14 Étant donné le nombre de textes, cette étude ne pouvait se baser que sur un échantillon, suffisamment représentatif pour permettre d'en tirer des conclusions générales. Le corpus devait donc rendre compte des multiples facettes de l'activité de traduction dans le milieu hagiographique médiolatin et des différentes phases de son évolution. Par conséquent, il devait inclure des traductions ad verbum et des traductions ad sensum, des textes issus des différentes régions qui ont produit des traductions et des différentes époques qui ont marqué l'histoire de la traduction hagiographique, des textes anonymes et des textes signés. Il devait aussi compter des textes peu ou pas du tout étudiés à côté de textes étudiés de manière plus ou moins approfondie; un des buts étant de parvenir, à travers la caractérisation linguistique des textes, à définir des manières de traduire, des traditions localisées, qui pourraient aider à situer dans le temps ou dans l'espace les textes sur lesquels on ne possède que peu d'information. Dans la mesure du possible, plusieurs témoins ont été choisis pour représenter chaque catégorie (type de traduction, époque, lieu, auteur). De même, ont été ajoutés à la liste certains textes pour lesquels il existe deux traductions ou bien une traduction et une réécriture, susceptibles de fournir eux aussi des informations sur les techniques de traduction. La sélection devait ainsi permettre plusieurs types de comparaisons et servir à faire ressortir les facteurs déterminants dans la classification des textes: évolution diachronique, spécificités régionales, spécificités d'écriture propres à une école de traduction ou à un traducteur, etc.

Dans la pratique, la sélection a dépendu de contraintes matérielles importantes. Elle a été réalisée dans les limites du matériel disponible et dans l'état actuel de nos connaissances, état qui ne peut être que provisoire, vu le nombre de textes encore inédits et celui de légendiers qu'il reste à dépouiller. Parmi les textes publiés, les rares qui ont fait l'objet d'une étude approfondie avec examen des différentes versions, comme la Passio Febroniae ( ChIEsA, 1990), la Passio Pelagiae (PetitMengin, 1981) ou la Passio Anastasii Persae (vircillo FRANKLIN, 2004), ont été choisis en premier. La priorité a ensuite été donnée aux éditions critiques, puis aux éditions diplomatiques et aux éditions basées sur un petit nombre de manuscrits, comme celle de Mombritius. Pour étoffer le corpus et permettre des comparaisons intéressantes entre deux traducteurs ou entre deux versions, il a fallu parfois ajouter d'autres textes édités de manière moins satisfaisante ${ }^{6}$. Ces contraintes ont donc ajouté à la sélection un côté aléatoire, limité mais inévitable, étant donné le caractère expérimental de la recherche.

Le corpus se compose de 35 traductions latines de Vies de saints et de Passions de martyrs réalisées en Italie, puisque c'est là que s'est concentrée l'activité de traduction hagiographique au Moyen Âge. Il compte des traductions anciennes, anonymes et très 
littérales, sans doute réalisées, pour la plupart, à Rome entre le $\mathrm{VI}^{\mathrm{e}}$ et le début du $\mathrm{IX}^{\mathrm{e}}$ siècle; des traductions romaines et napolitaines signées par différents hagiographes des $\mathrm{IX}^{\mathrm{e}}$ et $\mathrm{X}^{\mathrm{e}}$ siècles, au style plus libre ; des traductions vraisemblablement issues d'Italie du Nord, non datées, anonymes et assez littérales; d'autres venant du Sud, dont certaines probablement composées au $\mathrm{XI}^{\mathrm{e}}$ siècle dans la colonie amalfitaine installée à Constantinople; quelques cas connus de réécriture de traduction.

Les textes sélectionnés sont : Passio Abbibi (BHL 8b, 7477b), 2 versions de la Vita Amphilochii (codex de Mantoue et codex de Würzburg), Passio Anastasiae (BHL 404), 2 versions de la Passio Anastasii Persae (BHL 410b et BHL 411a), Passio Barbari (BHL 971d), 2 versions de la Passio Blasii (BHL 1370 et BHL 1380-1379), 2 versions de la Passio Catherinae (BHL 1657 et BHL 1659), Passio Eleutherii (BHL 2451), Vita Euphrosynae (BHL 2722), Passio Eustachii (BHL 2761b), Passio Eustratii (BHL 2778), 2 versions de la Passio Febroniae (BHL 2843 et BHL 2844), Vita Gregorii Thaumaturgi (BHL 3677m, 3678, 3678d), Passio Hermuli (BHL 3858b), 2 versions de la Vita Iohannis Calybitae (BHL 4358 et la version de P. Chiesa), Passio Luciae (BHL 4992), 2 versions de la Vita Mariae Aegyptiacae (BHL 5415 et BHL 5417), Passio Marini pueri (BHL 5538), 3 versions de la Passio Pelagiae (BHL 6604t et sa réécriture BHL6605, BHL 6609b), 2 versions de la Passio Petri Alexandrini ep. (BHL 6692-6693 et BHL 6698b), Passio Romani et soc. (BHL 7299b), 2 versions de la Passio XL martyrum Sebastenorum (BHL 7539 et BHL 7540), Poenitentia Theophili (BHL 8121) et sa réécriture Historia Theophili (BHL 8122).

\section{Hyperbase}

18 Après avoir été lemmatisés et analysés, les textes ont été placés sous Hyperbase. $\mathrm{Ce}$ logiciel, développé à l'Université de Nice par Étienne Brunet, offre plusieurs possibilités d'interrogation et d'exploitation statistique $d u$ corpus. Plusieurs bases ont été enregistrées afin de permettre l'exploitation de différents sous-corpus. Les textes ont été introduits selon un ordre défini. D'habitude cet ordre répond à un critère chronologique (ce qui permet de repérer, dans certaines représentations, une évolution dans le temps). Dans le cas du corpus hagiographique, le critère chronologique n'était pas approprié, pas plus que le critère géographique ou le classement par auteur, vu le nombre de textes anonymes, non datés et non localisés. J'ai donc choisi de combiner les différents critères afin de rendre au mieux la complexité du corpus. J'ai commencé par distinguer les textes non traduits des traductions. J'ai placé en premier les deux œuvres de référence que sont la Vie de Martin et celle de Benôt, puis les textes de Gaule et d'Italie non traduits? Viennent ensuite les traductions, avec d'abord celles sur lesquelles on ne possède que peu d'informations (que j'ai choisi de regrouper sous l'appellation «orphelines $»^{8}$ ), puis les traductions bien localisées, issues de Rome, de Naples et d'Amalfi. Ce classement n'est pas parfait, mais il a l'avantage de présenter un intérêt plus grand qu'un simple classement alphabétique. Dans les analyses, les textes sont souvent considérés d'après le groupe auquel ils appartiennent. Il faudra donc garder à l'esprit le caractère un peu grossier de la répartition par groupes, rien n'empêchant d'ailleurs de considérer les textes séparément quand il le faut. Sur les graphiques, les textes sont désignés par le nom du saint, pour des raisons pratiques. Il sont éventuellement précédés d'un chiffre quand il y a plusieurs versions (le 1 étant donné à la version la plus ancienne). 
Traductions « orphelines »

- Sans localisation: 1 Blaise (BHL 1370), 1-Catherin (BHL 1657), Eleuthère (BHL 2451), Euphrosyne (BHL 2722), Eustache (BHL 2761b), Lucie (BHL 4992), 1_MarieEg (Marie 1'Egyptienne, BHL 5417), Marin (BHL 5538), Romain (BHL 7299b), 1_Sébaste (BHL 7539)

- Italie du nord : Barbare (BHL 971d), 1 Fébronie (BHL 2844), 1 Pélagie (A, BHL 6604t), 3_Pélagie (A', BHL 6605), 2_Pélagie (C, BHL 6609b), $\overline{2}$ _Théophil (BHL 8122)

- Italie du Sud : Anastasie (BHL 404), Hermule (BHL 3858b)

Traductions romaines

- 1 Anastase (Anastase le Perse, BHL 410b, attribuée à Théodore de Tarse)

- Abbibe (BHL 8b-7477b, signé par Leo)

- 2 Anastase (BHL 411a, signée par Grégoire)

- 3 traductions d'Anastase le Bibliothécaire: 1_Amphiloq (Mantoue, attribuée), 1_JCalybit (Jean Calybite, BHL 4358, signée), 1_PierreA (Pierre d'Alexandrie, BHL 6698b, attribuée)

Traductions napolitaines

- 2 traductions signées par Paul diacre : 1_Théophil (BHL 8121), 2-MarieEg (BHL $5415)$

- 3 traductions de Guarimpotus : 2_PierreA (BHL 6692-6693, attribuée), 2_Fébronie (BHL 2843, attribuée), Eustrate (BHL 2778, signée)

- 1 signée par Jean diacre : 2 Sébaste (BHL 7540)

- 1 signée par Bonitus : 2_Blaise (BHL 1380-1379)

- 2 signées par Pierre sous-diacre: Thaumaturg (Grégoire Thaumaturge, BHL $3677 \mathrm{~m}, 3678,3678 \mathrm{~d}), 2$ 2_Catherin (BHL 1659) Traductions amalfitaines

- 2_Amphiloq (Würzburg, attribuée à Jean d'Amalfi)

- 2 JCalybit

Classement des traductions sur Hyperbase

\section{Analyses}

Les analyses qui suivent ne représentent qu'une partie de celles qui ont été réalisées lors de ma thèse. Elles ont été choisies soit parce qu'elles apportent des résultats concrets à l'étude des traductions, soit parce qu'elles ouvrent de nouvelles pistes de recherche ; dans tous les cas, elles servent à montrer les potentialités et l'efficacité des méthodes quantitatives. Cette enquête, qui s'est voulue originale sur le plan méthodologique, démontre en effet l'intérêt de les utiliser dans le domaine hagiographique, même pour un sujet aussi complexe que celui-ci, et à les considérer comme un outil supplémentaire et complémentaire, qui ne se substitue en rien au travail philologique traditionnel; les tests statistiques ne pouvant d'ailleurs être interprétés correctement que par un retour systématique aux textes.

Dans les analyses, le corpus est d'abord étudié dans son ensemble, face au corpus de textes non traduits, afin de dégager les caractéristiques des traductions en général. Il est ensuite envisagé seul, puis en différents sous-corpus, afin de faire ressortir les spécificités de chaque groupe de traductions. Les textes sont comparés sur plusieurs paramètres, lexicaux et syntaxiques, et au moyen de divers tests statistiques, qui vont du calcul général de la distance intertextuelle à l'examen plus précis de la distribution d'un mot ou d'une catégorie en particulier?.

\section{Distance lexicale}

21 Grâce à Hyperbase, il est possible de mesurer la distance, éloignement ou proximité, entre les textes en fonction du vocabulaire qu'ils partagent et du vocabulaire spécifique à 
chacun d'eux. Cette analyse est basée sur le calcul binomial de C. Muller, ajusté par un algorithme de D. LABBÉ (2000). Elle présente l'avantage de tenir compte de la distribution des fréquences et pas seulement de la présence ou de l'absence d'un mot; elle donne ainsi une meilleure appréciation de la distance intertextuelle, puisque c'est la surface totale des textes qui est examinée et pas seulement leur lexique. Le tableau de distribution obtenu peut être traité par le procédé de l'analyse factorielle des correspondances ou par celui de l'analyse arborée.

\section{Corpus global}

La distance lexicale a d'abord été mesurée entre tous les textes du corpus (traductions et non traductions $)^{10}$ au moyen de l'analyse factorielle. Les résultats sont représentés par le graphique qui suit. L'interprétation du graphe se fait en considérant d'une part la distance entre les points et d'autre part leur distribution par rapport aux axes. Les ensembles dessinés représentent, de façon un peu grossière, les différents groupes ; il n'y a pas de véritable intersection entre les cercles.

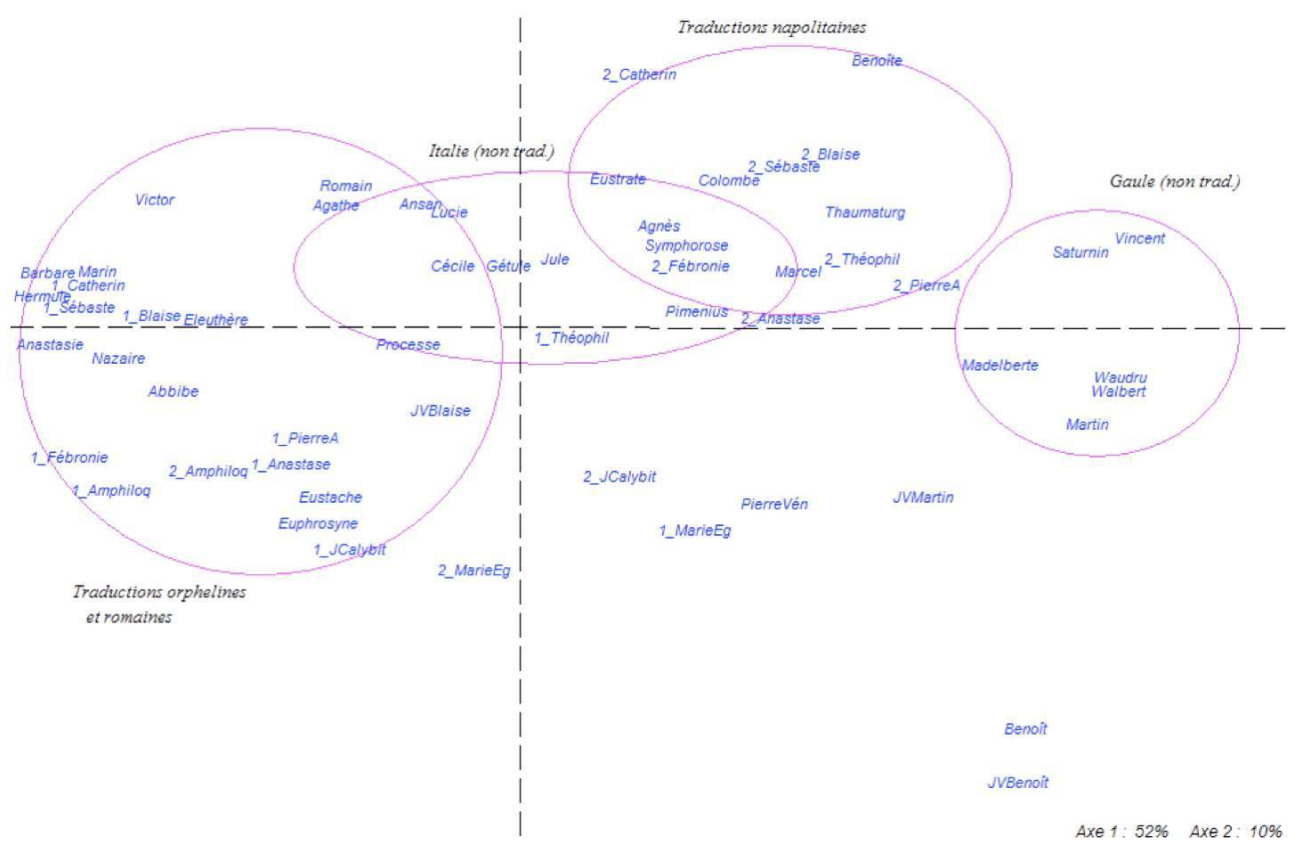

Analyse factorielle de la distance lexicale à travers le corpus global

Dans cette analyse, les deux premiers facteurs cumulés rendent compte de $62 \%$ de l'information contenue dans le tableau de distribution. Le premier, qui exploite la plus grande part de données $(52 \%)$ et qui est représenté par l'axe horizontal, distingue nettement les textes de Gaule et les traductions napolitaines, à droite, des traductions orphelines et romaines, à gauche. Le deuxième facteur (représenté par l'axe vertical) s'attache surtout à la Vie de Benoît de Grégoire le Grand et à son abbreuiatio par Jacques de Voragine, dont la position isolée dans le cadran inférieur droit (aussi bien par le premier facteur que par le deuxième) souligne l'originalité lexicale rapport au reste du corpus. Les autres textes italiens qui ne sont pas des traductions occupent le centre du graphique, surtout au-dessus de l'axe horizontal.

Le lexique mesuré en fonction de sa distribution ne semble donc pas être, pour ce corpus, un critère suffisant pour distinguer les traductions des textes non traduits. Par contre, en 
ce qui concerne les traductions, il fait apparaître des regroupements et des oppositions très intéressants, que d'autres analyses vont préciser.

\section{Sous-corpus italien}

La distance lexicale a été recalculée dans un sous-corpus constitué exclusivement des textes issus d'Italie, certains traduits, d'autres pas. Le retrait des textes de Gaule se justifie par leur position écartée (à l'extrémité droite sur le graphique précédent), qui traduit leur éloignement et leur spécificité lexicale : les retirer permet d'éliminer le poids de ces textes que l'on sait différents et de limiter en même temps le facteur géographique à l'Italie. À l'intérieur du sous-corpus italien, la distance a été mesurée au moyen de l'analyse arborée de X. Luong (LUONG, 1994; BARTHELEMY, LUONG, 1998). Les résultats sont représentés sous la forme d'un arbre, avec des branches, des rameaux et des nœuds. Tous les textes sont liés les uns aux autres, par des chemins plus ou moins directs, qui reproduisent les distances. C'est donc la longueur cumulée des segments qui unissent un texte à un autre qui doit être considérée (la distance à vol d'oiseau n'a aucune importance).

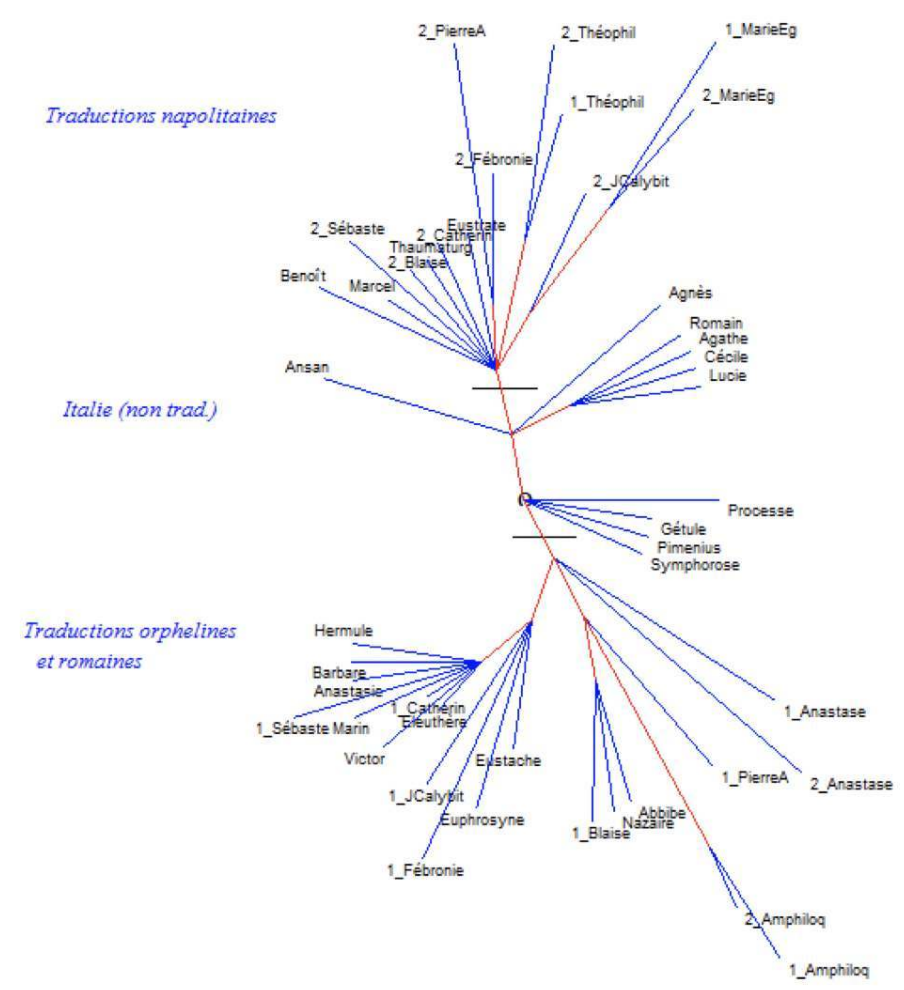

Analyse arborée de la distance lexicale à travers le sous-corpus italien

L'analyse arborée fait apparaître plus clairement encore les oppositions et les regroupements dans le corpus. Elle vient corroborer les premières observations. Les traductions napolitaines, groupées à la cime de l'arbre, s'opposent nettement aux traductions orphelines et romaines, situées à l'autre extrémité. Les textes italiens qui ne sont pas des traductions se situent entre les deux, dans une zone d'intermédiarité mal structurée, qui montre tout de même qu'ils se distinguent des traductions en ce qu'ils semblent relativement indifférents à ce critère d'opposition. Les regroupements et les oppositions observés ne répondent donc pas à la question «traduction ou non 
traduction", mais semblent plus sensibles aux facteurs géographiques et/ou chronologiques, ainsi qu'au mode de traduction et au niveau rédactionnel.

La plupart des traductions orphelines sont rassemblées en bas de l'arbre sur une ramification à gauche. Au bout de celle-ci, les textes sont groupés en un amas de petites branches serrées qui montrent leur proximité. Un deuxième groupe se trouve un peu plus haut: la longueur des rameaux montre que la distance qui les sépare est un peu plus grande que dans le premier groupe. Seules les traductions des Passions de Lucie et de Romain se trouvent au centre de l'arbre, rattachées aux Passions d'Agathe et de Cécile.

28 À l'extrémité basse de la branche principale, on trouve les deux Vies d'Amphiloque à courte distance l'une de l'autre. Leur proximité prouve leur connexion lexicale, et leur situation sur une longue branche à l'écart montre leur spécificité par rapport aux autres textes du corpus. Un peu plus haut, les deux Passions d'Anastase le Perse se rejoignent aussi, mais avec une plus grande distance entre elles.

Du côté napolitain, la connexion entre les textes est très forte également, même si certains textes sortent du lot. La longueur des rameaux à droite révèlent la spécificité des textes de Paul diacre, la Vie de Marie l'Égyptienne et la Pénitence de Théophile, toutes deux liées à une version qui leur est apparentée. Les deux textes attribués à Guarimpotus, la Passion de Fébronie et la Passion de Pierre d'Alexandrie, se trouvent eux aussi sur une branche distincte, tandis que la Passion d'Eustrate, signée par Guarimpotus, est directement attachée au reste du groupe. La connexion lexicale qui existe dans le groupe napolitain apparaît plus forte qu'avec le reste du corpus, y compris les versions qui leur sont apparentées thématiquement (comme les traductions orphelines de la Vie de Catherine d'Alexandrie, des Passions de Fébronie, de Blaise et des martyrs de Sébaste, ou la Passion anastasienne de Pierre d'Alexandrie).

30 La structure de l'arbre vient confirmer ce fait. En donnant l'ordre dans lequel se sont créés les nœuds qui relient les branches, elle indique la force des regroupements entre les éléments. Dans cet arbre, les chiffres montrent que les premiers nœuds, donc les connexions les plus fortes, concernent les Vies de Marie l'Égyptienne, les Pénitences de Théophile, les Vies d'Amphiloque, puis les traductions napolitaines - d'abord les Passions de Blaise et des martyrs de Sébaste, puis les Vies de Grégoire le Thaumaturge et de Catherine écrites par Pierre sous-diacre, enfin les textes traduits par Guarimpotus, les Passions de Pierre et de Fébronie, puis d'Eustrate, qui se rattache aux autres.

\section{Facteur thématique}

31 Le facteur thématique a évidemment beaucoup de poids dans une analyse lexicale. En témoigne ce nouvel arbre, qui illustre la distance lexicale à l'intérieur d'un autre souscorpus, composé exclusivement des traductions et des réécritures. 


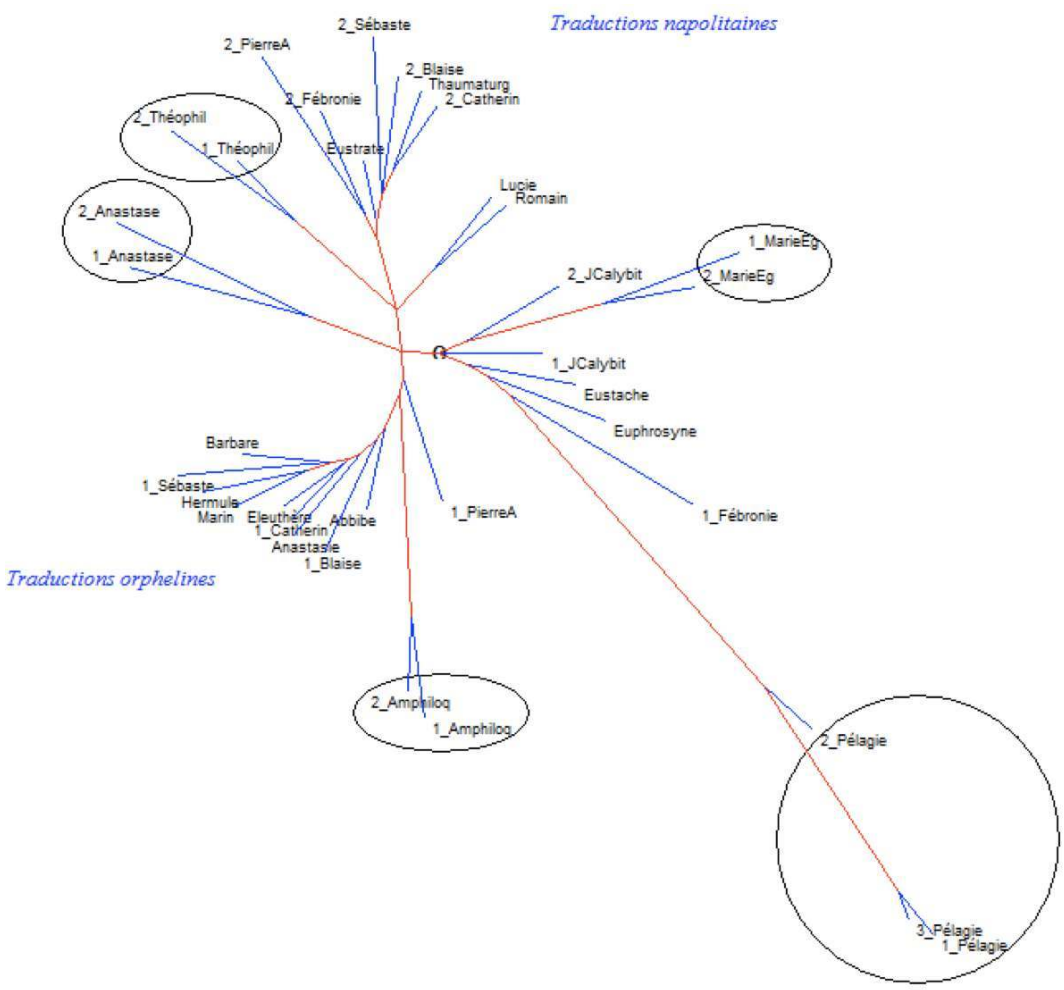

Analyse arborée de la distance lexicale à travers le sous-corpus de traductions

Cet arbre, qui corrobore lui aussi l'opposition entre les traductions orphelines, en bas, et les traductions napolitaines, en haut (qui se trouvent en outre rangées ici en ordre chronologique avec, en remontant, les textes de Guarimpotus, de Jean diacre, de Bonitus et de Pierre sous-diacre - ceux de Paul diacre étant toujours à part), montre plusieurs regroupements de versions apparentées par le sujet (les 2 Pénitences de Théophile et les 2 Vies de Marie, les 2 Passions d'Anastase, les 2 Vies d'Amphiloque, les 3 Passions de Pélagie, avec une connexion plus forte entre la traduction la plus ancienne et sa réécriture), prouvant le poids du facteur thématique. Mais en même temps, l'arbre montre que, même si ce facteur pèse lourd dans le calcul de la distance lexicale, il ne prévaut pas dans tous les cas : en effet, les Passions de Fébronie, de Catherine, de Pierre, de Blaise, et des martyrs de Sébaste issues de Naples restent ici aussi opposées aux traductions anciennes et romaines qui leur sont apparentées.

D'autres facteurs interviennent donc dans la classification des textes, parfois plus puissants que le facteur thématique. Si celui-ci est facilement identifiable, les autres facteurs susceptibles d'intervenir ne le sont pas autant. Dans le calcul de la distance lexicale, c'est en effet tout le vocabulaire qui est examiné et la distribution de tous les mots qui est comparée à travers tout le corpus ou sous-corpus étudié. Or, le vocabulaire d'un texte s'explique de diverses manières: le genre, le thème, le style, mais aussi l'époque, la région, les spécificités d'une école, les caractéristiques personnelles d'un auteur (les mots qu'il aime utiliser, les tics de langage, les petits mots-outils qu'il emploie sans faire attention, etc.). Il n'est pas toujours possible de déterminer avec exactitude quels facteurs interviennent, ni de mesurer leur poids respectif, tant ils sont parfois entremêlés. Dans l'examen du corpus de traductions, certaines connexions apparaissent très fortes et se maintiennent tout au long des analyses, d'autres se modifient. Ces modifications, qui prouvent elles aussi que plusieurs facteurs peuvent intervenir, 
indiquent en même temps que ce ne sont pas toujours les mêmes qui prédominent et que leur influence peut varier selon les corpus considérés (la distance étant recalculée pour chaque nouveau corpus).

Par exemple, si on retire les deux cas connus de réécriture du corpus de traductions (l'Histoire de Théophile et une Passion de Pélagie), l'analyse arborée révèle des regroupements qui n'apparaissaient pas précédemment, ou pas de manière aussi manifeste. Outre l'originalité des traductions napolitaines qui s'est manifestée dès le départ, et leur opposition aux traductions orphelines, outre la proximité de versions thématiquement apparentées, l'analyse de ce nouveau sous-corpus rapproche en plus des textes qui sont historiquement liés. On voit en effet que, si les 2 Vies de Marie et les 2 Passions de Pélagie restent ensemble en raison du facteur thématique, les 2 Passions d'Anastase le Perse sont à présent séparées, ainsi que les 2 Vies d'Amphiloque. La première version de la Vie d'Amphiloque rejoint la branche de son auteur, Anastase le Bibliothécaire, auprès des autres traductions dont il s'est chargé (la Vie de Jean Calybite et la Passion de Pierre d'Alexandrie), tandis que la deuxième version de la Vie d'Amphiloque se trouve à présent sur la même branche que la deuxième version de Jean Calybite, probablement rédigée comme elle à Amalfi. La Pénitence de Théophile par Paul diacre n'est plus attirée par sa réécriture et rejoint directement la branche qui porte les deux versions de la Vie de Marie l'Égyptienne, dont l'une est l'œuvre de Paul diacre. La position de cette branche située plus bas que les autres branches napolitaines, plus proche des traductions romaines d'Anastase le bibliothécaire et des traductions orphelines, souligne encore un peu plus la particularité des œuvres de Paul diacre, premier traducteur connu de l'École, au style encore très littéral. Du côté napolitain toujours, on remarque que la Passion de Pierre et la Passion de Fébronie, les deux œuvres attribuées à Guarimpotus, restent sur la même branche, pas loin de la Passion d'Eustrate signée par lui, tandis que les deux œuvres signées par Pierre sous-diacre, la Passion de Catherine et la Vie de Grégoire le Thaumaturge, qui étaient réunies avant, sont à présent séparées. La longueur de la branche qui réunit la Passion de Blaise à la Passion des martyrs de Sébaste souligne à la fois la connexion qui existe entre ces deux textes liés par le sujet et leur originalité lexicale.

Le cas de la Passion d'Anastase le Perse est un peu différent. Dans cette nouvelle analyse, la traduction ancienne, très littérale, a rejoint le côté des traductions orphelines, et plus précisément la branche qui réunit la Passion d'Hermule à la Passion d'Anastasie en bas de l'arbre, deux traductions orphelines très littérales, originaires du Sud de l'Italie. L'autre version est restée du côté napolitain, avec lequel elle montre d'autres affinités, comme on le verra. 


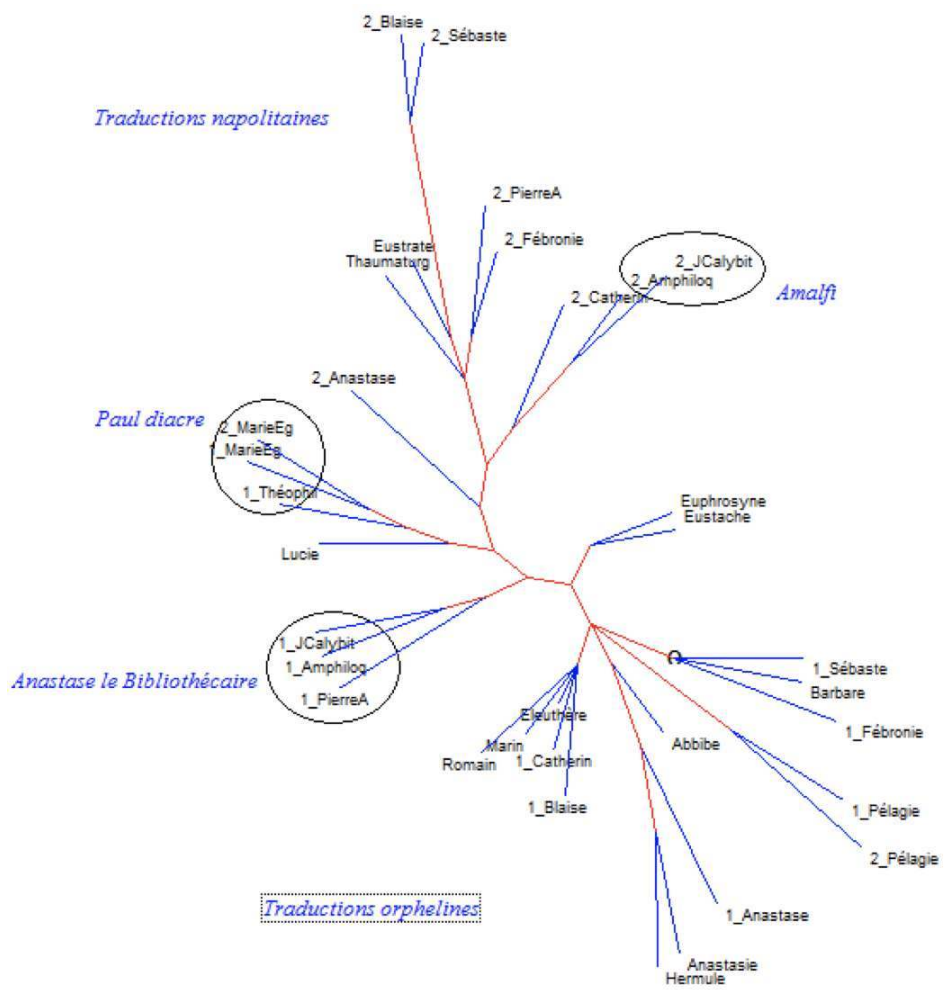

Analyse arborée de la distance lexicale à travers le sous-corpus de traductions réduit l'originalité des traductions napolitaines qui est apparue. Leur opposition systématique aux autres traductions ainsi que leur forte connexion soulignent leur grande spécificité. Il est connu que les traductions issues du cercle napolitain sont de meilleure qualité et rédigées dans un style généralement plus élevé que la majorité des traductions médiolatines. La distinction est donc attendue sur le plan syntaxique, ces analyses prouvent qu'elle se manifeste aussi au niveau lexical.

\section{Originalité lexicale de l'école napolitaine}

La question suivante est de savoir ce qui, dans le vocabulaire, peut les distinguer des autres traductions du corpus de manière aussi manifeste. Elles doivent en effet partager une part importante de vocabulaire, qui doit être assez spécifique pour les distinguer à tel point des autres traductions, et singulièrement des versions qui ont la même thématique. Qu'est-ce qui les caractérise donc ? Y a-t-il vraiment un vocabulaire spécifique, propre à l'école ? Un examen minutieux des listes de vocabulaire serait nécessaire pour répondre à ces questions. Une autre possibilité d'obtenir des éléments de réponse est de travailler à partir des listes de spécificités que propose Hyperbase. Par un calcul de l'écart réduit, il est en effet possible de connaître le vocabulaire spécifique d'un texte, c'est-à-dire le vocabulaire qui y est employé de manière significative par rapport au reste du corpus. Il 
reste à comparer ces listes, afin de comprendre ce qui fait l'originalité lexicale du groupe. Je présenterai deux cas de versions apparentées par le sujet et issues de groupes différents, en prenant parmi les versions napolitaines deux textes attribués à un seul et même auteur : la Passion de Pierre d'Alexandrie attribuée à Guarimpotus et celle d'Anastase le Bibliothécaire, puis la Passion de Fébronie attribuée au même Guarimpotus et la version anonyme probablement traduite dans le Nord de l'Italie.

Dans la Passion napolitaine de Pierre d'Alexandrie, les premiers lemmes employés de manière significative par rapport au reste du corpus sont Arius, haereticus et Arianus. Arius est utilisé 25 fois par Guarimpotus et 8 fois par Anastase. Haereticus est employé 12 fois et Arianus 7 fois par Guarimpotus, alors que tous deux sont absents chez Anastase. Ces premiers mots indiquent une des thématiques de l'œuvre du Napolitain, l'hérésie arienne, un sujet qui lui tient visiblement à cœur et qui le pousse à de longs développements ( TELFER, 1949). On relève de même, un peu plus loin sur la liste, catholicus, blasphemia, persecutio, scindo (employé 3 fois au sujet de l'Église), vesania, perversus, etc. Arius intervient dans la version anastasienne, mais il n'occupe pas la même place que chez Guarimpotus. La liste du vocabulaire spécifique dans la version d'Anastase montre en effet que les lemmes les plus significatifs sont des termes attachés à des réalités plus concrètes de l'histoire de Pierre : populus, Petrus, tribunus, sedes, archiepiscopus, thronus, etc.

En ce qui concerne la Passion de Fébronie, dans les deux versions, les premiers mots spécifiques, après les noms propres, sont relatifs à l'histoire et ne renvoient pas à des réflexions personnelles. La version septentrionale semble un peu plus attachée au martyre de Fébronie, comme le suggère la présence des mots miles, excido, fugio, auditorium. Dans la version napolitaine, on relève néanmoins le verbe très expressif detrunco, employé à 5 reprises, et on note qu'il n'est attesté qu'une seule autre fois dans tout le corpus et que c'est justement dans la Passion de Pierre d'Alexandrie de Guarimpotus. Dans les deux versions, on trouve des mots qui n'apparaissent dans aucune autre traduction du corpus, comme nato ${ }^{11}$, pauso, avunculus, glosochomum dans la version septentrionale ou asceterium et palaestra dans la napolitaine. Paolo CHIESA, qui a édité les deux versions (1990: 276-288), a mis en évidence, chez Guarimpotus, la recherche de variation et de synonymes, l'introduction de termes et d'expressions complexes. Il note par exemple que, pour traduire $\sigma \tau \rho \alpha \tau \imath \tilde{\omega} \tau \alpha \mathrm{l}$, il emploie bien entendu milites, mais aussi apparitores, satellites et carnifices, qui se trouvent aussi sur la liste des spécificités. Le mot apparitor est attesté 10 fois dans le corpus italien, dont 7 dans le groupe napolitain ( $1 \mathrm{ici}, 3$ dans la Passion d'Eustrate signée Guarimpotus et 3 dans la Passion de Blaise). Satelles est attesté 4 fois dans le corpus et uniquement dans les textes de Naples (dont 2 ici). Pour

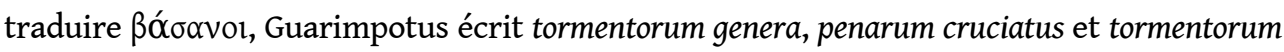
patibula. Le mot cruciatus est attesté 16 fois dans le corpus italien, dont 11 dans les traductions, et, parmi ces 11 occurrences, 9 dans les traductions de Naples (dont 5 chez Guarimpotus : 3 ici, 1 dans la Passion de Pierre et 1 dans celle d'Eustrate). Pour $\theta \varepsilon o ́ c$, il écrit omnipotentia divine maiestatis ou sanctam et individam trinitatem; pour le pluriel, il emploie deux fois deorum numina. Numen est attesté 6 fois dans le corpus italien, dont 5 fois dans les traductions napolitaines ( 2 ici et 3 chez Pierre sous-diacre).

41 Une part de l'originalité lexicale des traductions napolitaines se laisse donc appréhender à travers l'étude de deux versions de Guarimpotus. Le premier exemple montre qu'une part du vocabulaire spécifique de Guarimpotus lui vient des digressions par lesquelles il évoque un problème qui lui tient à cœur, l'hérésie arienne, et qui est moins présent dans 
le texte d'Anastase. Le second exemple met en évidence l'emploi d'hellénismes d'un côté comme de l'autre, mais aussi la recherche de mots rares et d'expressions complexes chez Guarimpotus. Il montre en outre que certains de ces mots rares se retrouvent dans d'autres traductions napolitaines alors qu'elles sont absentes du reste du corpus.

\section{Distance grammaticale}

La distance entre les textes, leur proximité ou leur éloignement, en d'autres termes leur ressemblance ou leur dissemblance, peut aussi être mesurée du point de vue de la syntaxe. Ce calcul est rendu possible grâce à l'étiquetage morphosyntaxique du corpus, qui permet de dénombrer tous les éléments constitutifs des textes (substantifs, adjectifs, verbes, mais aussi substantifs de la première déclinaison, adjectifs de la deuxième classe, verbes de la troisième conjugaison, etc.) et de connaître, en même temps, la distribution des cas et des nombres des substantifs, la distribution des temps et des modes des verbes dans les principales et dans les subordonnées, etc. Il s'agit dès lors de voir s'il est possible de regrouper les textes ou de les différencier en fonction de la manière dont s'y distribuent les catégories grammaticales ou les structures syntaxiques (parties du discours, types de subordonnées, temps et modes verbaux, cas nominaux, etc.). Hyperbase propose un calcul général pour mesurer la distance grammaticale en prenant en compte la distribution et la répartition de tous les codes grammaticaux à travers le corpus. Des analyses plus ciblées peuvent ensuite affiner les résultats, en examinant certaines distributions en particulier et à travers des sous-corpus spécifiques.

\section{Corpus global}

Le premier graphique illustre la distance grammaticale dans le corpus global ${ }^{12}$ d'après la distribution de tous les codes grammaticaux. Cela signifie que l'analyse complète de chaque forme a été prise en considération: les substantifs de la première déclinaison employés au nominatif singulier ont été distingués des substantifs de la première déclinaison employés au nominatif pluriel, les indicatifs présents actifs des verbes de la troisième conjugaison employés à la première personne du singulier ont été distingués des indicatifs présents actifs des verbes de la troisième conjugaison employés à la deuxième personne du singulier ou à la première du pluriel, et ainsi de suite. 


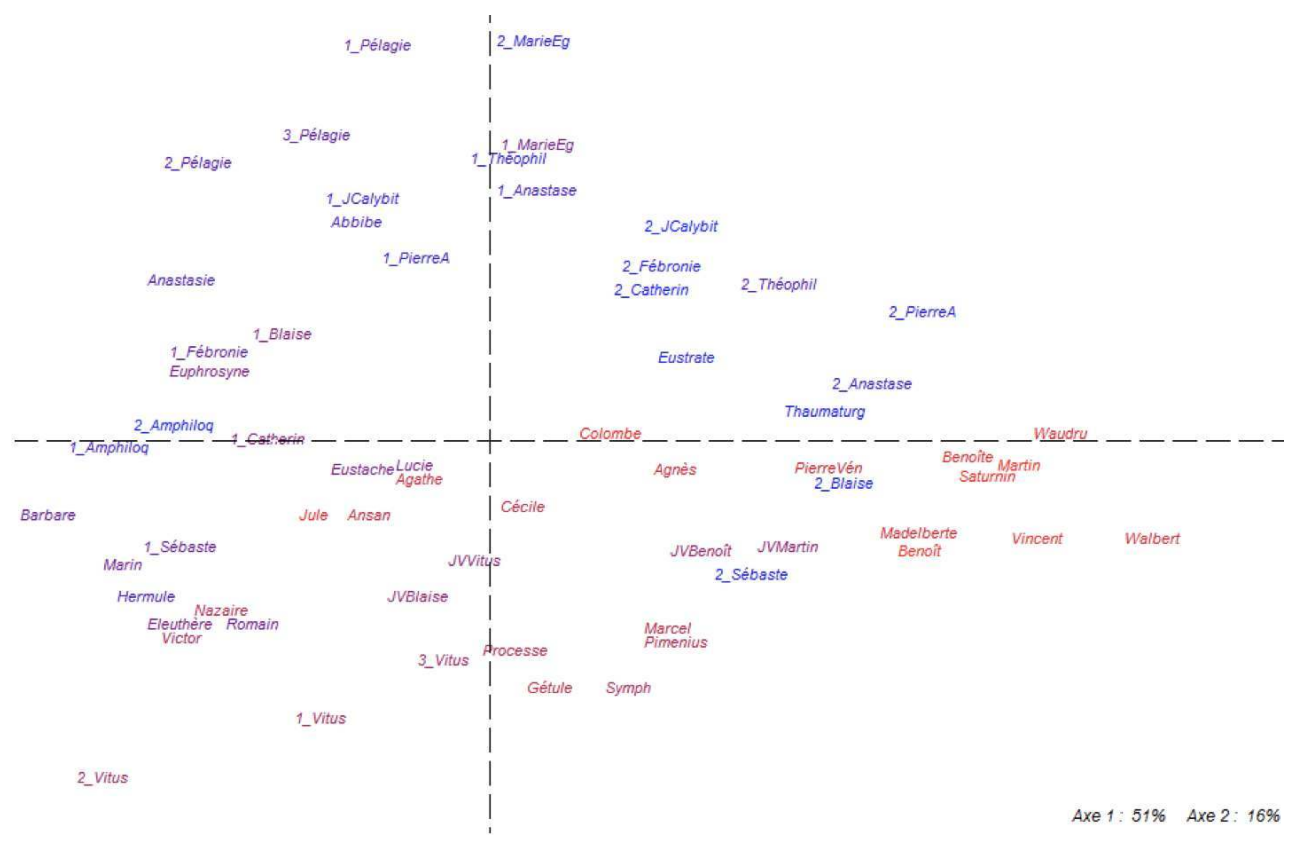

Analyse factorielle de la distance grammaticale à travers le corpus global

Les deux premiers facteurs accaparent $67 \%$ de l'inertie : le premier en utilise $51 \%$ et le deuxième $16 \%$. Le premier facteur s'attache principalement aux textes de Gaule, qu'il situe à l'extrémité droite, soulignant ainsi leur originalité sur le plan grammatical aussi. Il regroupe ensuite toutes les traductions napolitaines à droite, principalement en haut, qu'il oppose aux autres traductions rassemblées à gauche. Quant au deuxième facteur, il permet de regrouper grosso modo les textes italiens non traduits en bas, plus ou moins près de l'axe vertical. Les couleurs affectées aux textes évoluent du rouge au bleu en fonction de l'ordre dans lequel les textes ont été introduits dans Hyperbase. On remarque qu'elles progressent en spirale, en partant du cadran inférieur droit vers la gauche en bas, puis en haut, pour revenir ensuite à droite, reflétant ainsi une évolution entre les différents groupes.

L'analyse arborée confirme la répartition par groupes. 


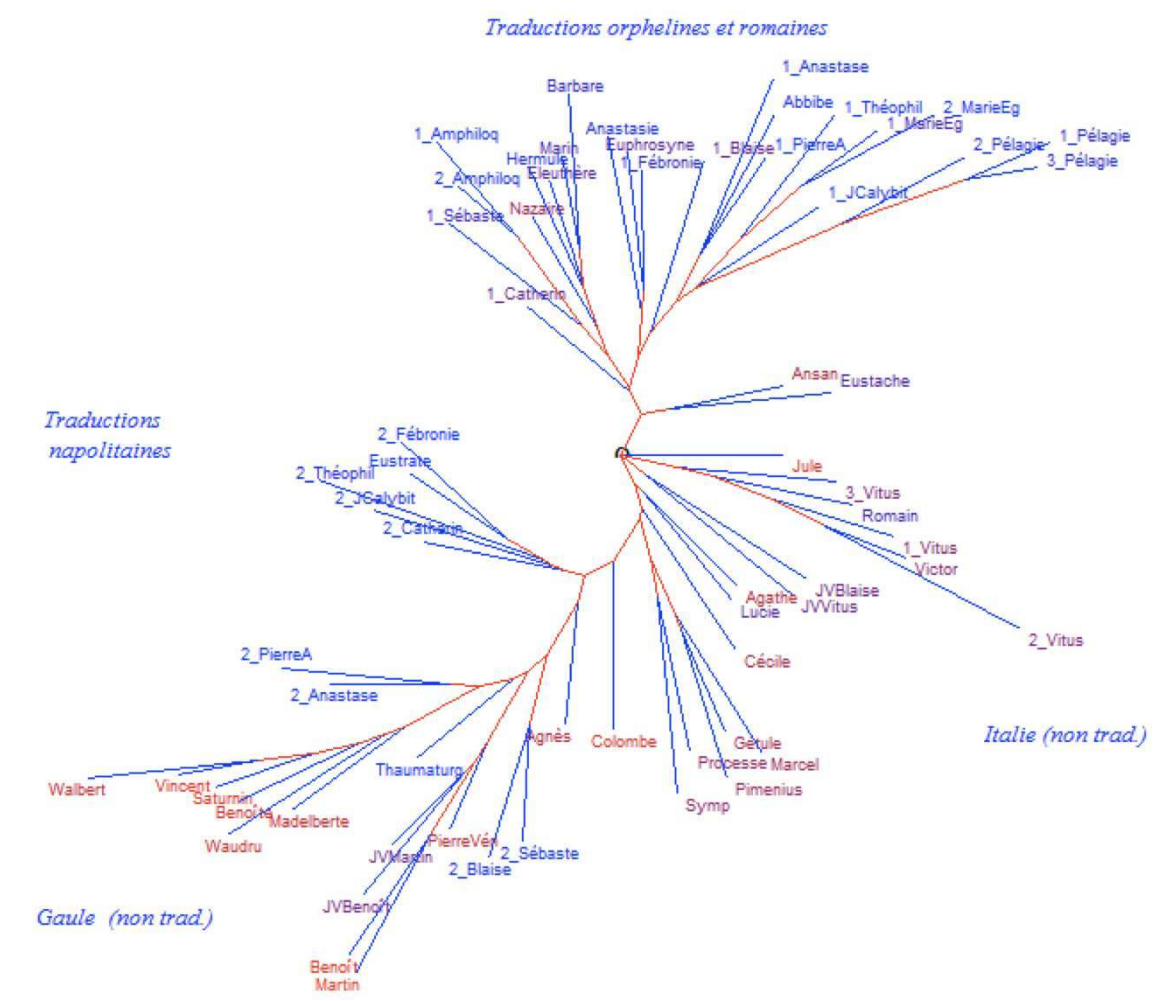

Analyse arborée de la distance grammaticale à travers le corpus global branches, prouvant ainsi leur connexion et leur spécificité grammaticale. Le bas de l'arbre porte aussi les traductions napolitaines et les textes italiens non traduits. Parmi les traductions napolitaines, la Passion de Pierre et la Vie de Grégoire le Thaumaturge, qui étaient situées un peu plus à droite sur le graphique de l'analyse factorielle, montrent ici aussi leur particularité en se situant plus près des textes de Gaule (la deuxième traduction de la Passion d'Anastase accompagne celle de Pierre). Il en va de même pour les Passions de Blaise et des martyrs de Sébaste, qui étaient passées sous l'axe horizontal de l'analyse factorielle : leur position sur une branche distincte prouve la forte connexion qui les unit et qui, en même temps, les distingue des autres traductions napolitaines sur le plan grammatical. Celles-ci sont situées un peu plus haut, à gauche, sur une autre ramification bien compacte, qui lie les Passions de Fébronie, d'Eustrate et de Catherine (auxquelles se joignent la réécriture de Théophile et la traduction amalfitaine de la Vie de Jean Calybite, comme dans l'analyse factorielle). Il reste les deux textes napolitains de Paul diacre. Le calcul de la distance lexicale avait montré leur particularité par rapport aux autres textes de Naples; l'examen des codes grammaticaux semble conduire à la même conclusion: les deux textes sont situés tout en haut de l'arbre, du côté des traductions romaines (les deux Vies de Marie sont réunies, comme dans l'analyse lexicale, mais la Pénitence de Théophile est séparée de sa réécriture).

Après les textes de Gaule et les traductions napolitaines, on trouve, vers le centre de l'arbre, les textes italiens non traduits, disposés sous la forme d'un éventail. Le haut de l'arbre porte, à gauche, les traductions orphelines assez bien regroupées, avec aussi les deux Vies d'Amphiloque ; à droite, les traductions romaines et les textes de Paul diacre, et, tout au bout, les trois Passions de Pélagie. La position de ces dernières montre une certaine spécificité sur le plan grammatical, moins importante toutefois que celle 
observée sur le plan lexical. La connexion est ici aussi plus forte entre la première traduction et sa réécriture.

Le calcul de la distance grammaticale à travers le corpus global fait donc apparaître différents groupes, qui, comme pour la distance lexicale, semblent correspondre aux grands groupes préalablement définis (textes de Gaule, textes d'Italie non traduits, traductions orphelines, romaines et napolitaines). En ce qui concerne les traductions, ce calcul met lui aussi en évidence l'originalité des textes napolitains.

\section{Sous-corpus italien}

L'analyse arborée du sous-corpus italien montre d'ailleurs une belle bipartition : avec, en gros, en haut de l'arbre les traductions orphelines et romaines et les textes de Paul diacre ; en bas, les traductions napolitaines et les textes italiens non traduits.

50 Le haut de l'arbre compte trois ramifications bien délimitées. Celles-ci étaient déjà présentes dans la représentation arborée du corpus global, mais elles apparaissent ici de façon beaucoup plus nette. La première regroupe les traductions orphelines, avec les sous-groupes observés; la deuxième porte trois traductions romaines (les traductions littérales des Passions d'Anastase le Perse et d'Abbibe, ainsi que la Passion anastasienne de Pierre), auxquelles se rattache celle de Blaise ; la troisième se divise en deux et montre d'une part la spécificité des trois Passions de Pélagie et le lien étroit qui unit la première traduction à sa réécriture, et d'autre part l'originalité des deux traductions de Paul diacre par rapport aux autres traductions napolitaines, avec une forte connexion entre les deux Vies de Marie (cette ramification porte aussi la traduction anastasienne de Jean Calybite).

51 Le bas de l'arbre est consacré aux textes italiens non traduits et aux traductions napolitaines. L'originalité des Passions de Vitus apparaît clairement. La Passion de Romain reste attachée à leur branche, tout comme la Passion de Lucie reste liée à celles d'Agathe et de Cécile. Du côté napolitain, la proximité des Passions de Blaise et des martyrs de Sébaste est confirmée, ainsi que leur distance par rapport aux autres. Les Passions d'Eustrate et de Fébronie restent elles aussi liées, comme celles de Pierre et d'Anastase (auxquelles s'ajoute l'Histoire de Théophile). Par contre, le nœud qui regroupe les deux traductions de Pierre sous-diacre (la Vie de Grégoire le Thaumaturge et la Passion de (atherine) avec la Vie amalfitaine de Jean Calybite et la Passion italienne d'Agnès est le dernier à se former, ce qui montre que ces textes sont moins bien intégrés dans le calcul. 


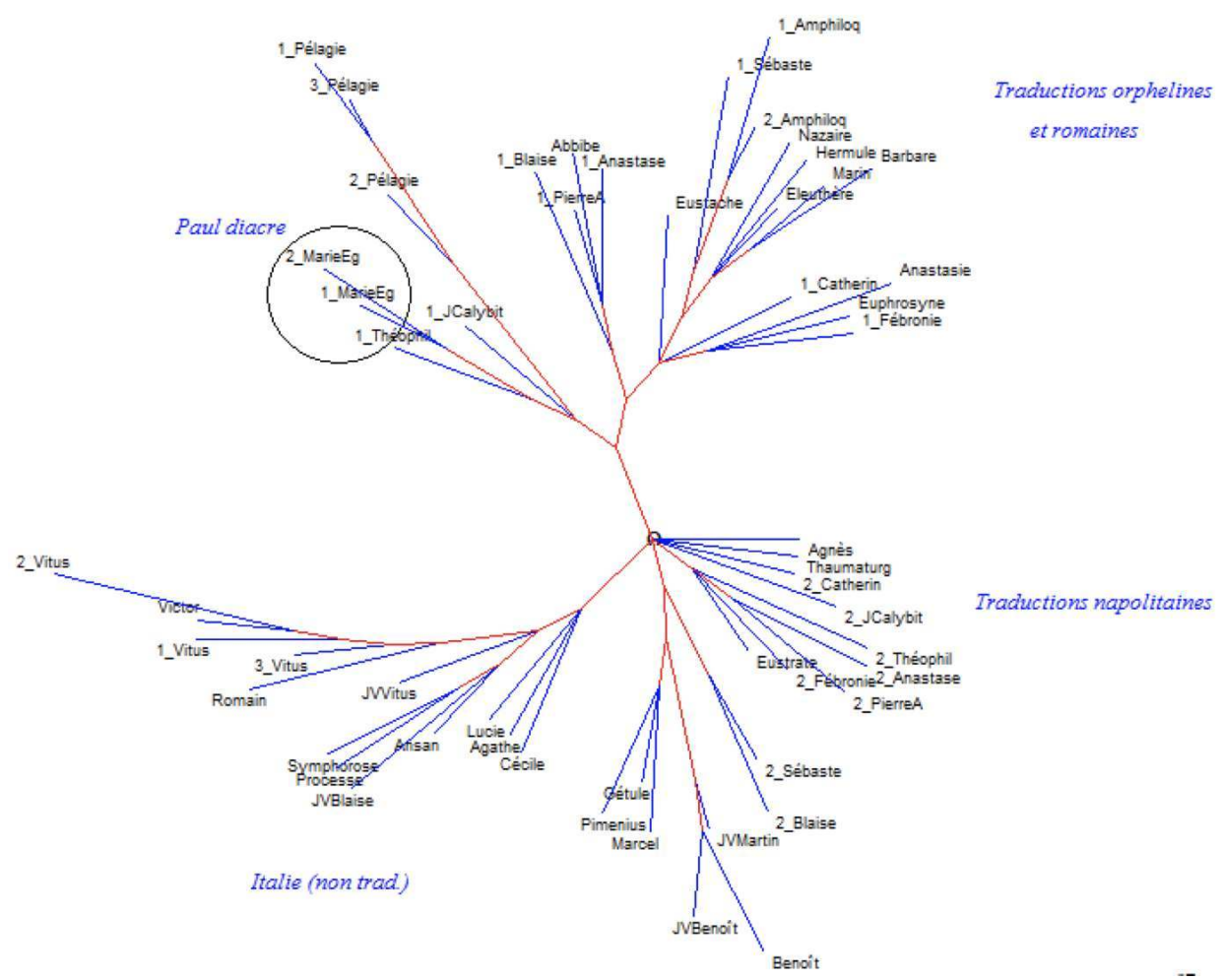

Analyse arborée de la distance grammaticale à travers le sous-corpus italien

Comme dans le calcul de la distance lexicale, force est de constater qu'il n'y a pas non plus une opposition nette entre les traductions et les textes non traduits sur le plan grammatical. Les textes non traduits sont bien regroupés, mais ils ne s'opposent pas franchement à un groupe qui rassemblerait toutes les traductions, même classées en différents sous-groupes. L'examen du corpus entier et du sous-corpus italien révèle l'existence de différents groupes de textes qui se maintiennent au fil des analyses et qui correspondent, à quelques exceptions près, aux groupes définis pour la constitution du corpus. Les textes non traduits forment un de ces groupes, distinct même s'il ne fait pas face à tous les autres réunis. L'opposition la plus évidente concerne à nouveau les traductions orphelines et les traductions napolitaines.

\section{Sous-corpus de traductions}

Une nouvelle analyse permet de préciser les rapports entre les traductions : le calcul de la distance grammaticale dans le sous-corpus constitué exclusivement des traductions. Le graphique de l'analyse factorielle donne une nouvelle illustration de l'opposition entre les traductions napolitaines, à gauche, et les traductions orphelines et romaines, à droite. Pour Naples, la position des deux textes de Paul diacre est ici aussi remarquable, tout en bas du deuxième axe et tendant vers les traductions romaines d'Anastase le Bibliothécaire. Dans le groupe des traductions orphelines, la situation écartée soit vers la gauche, soit vers le haut, des Passions de Lucie et de Romain confirme leur originalité sur le plan grammatical. 


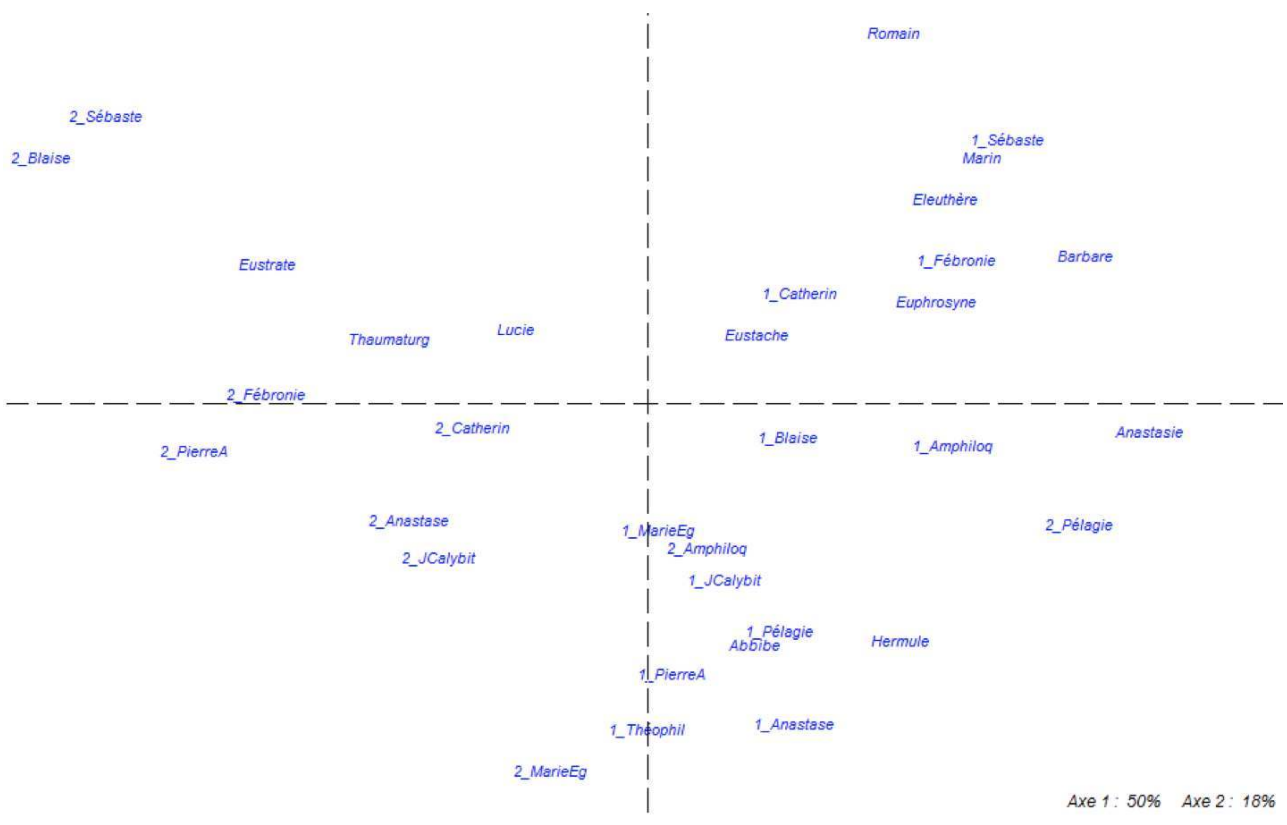

Analyse arborée de la distance grammaticale dans le sous-corpus de traductions

Dans ces analyses, la distance grammaticale a été mesurée d'après la distribution de tous les codes grammaticaux. Il est par conséquent difficile de savoir quel paramètre contribue à rapprocher ou à éloigner les textes. Des calculs plus ciblés sont donc nécessaires.

\section{Distribution des parties du discours}

On peut commencer par se demander comment les textes se situent les uns par rapport aux autres d'après l'utilisation qu'ils font des catégories grammaticales. Celles-ci sont-elles distribuées de manière identique dans tous les textes du corpus ou y a-t-il des emplois particuliers suffisamment significatifs pour caractériser un texte ou un ensemble de textes?

\section{Comparaison entre le corpus de traductions et le corpus de textes non traduits}

J'ai choisi d'aborder cette question de manière globale pour voir si les catégories grammaticales sont utilisées indifféremment ou non dans le sous-corpus de traductions et dans celui des textes non traduits. Dans un premier temps, les deux corpus sont donc comparés directement l'un à l'autre, sans distinction des textes qui les composent. Toutes les traductions sont regroupées sous l'appellation «corpus de traductions » et tous les textes non traduits sous celle de " corpus de comparaison ». Pour cette analyse, j'ai utilisé le calcul de l'écart réduit. Dans un tableau à deux colonnes, j'ai inscrit les effectifs de chaque catégorie ${ }^{13}$, puis formé l'hypothèse d'une répartition aléatoire, faite au prorata de la longueur de chaque corpus, et calculé les effectifs que l'on devrait avoir d'un point de vue théorique. La différence entre ces effectifs théoriques et les effectifs réellement observés est évaluée par le calcul de l'écart réduit. Un écart est considéré significatif s'il est supérieur à $2(+2,-2)$.

Effectifs observés : 


\begin{tabular}{|l|l|l|l|}
\hline & Corpus de traductions & Corpus de comparaison & TOTAL \\
\hline Substantifs & 37057 & 22481 & 59538 \\
\hline Adjectifs & 8255 & 4889 & 13144 \\
\hline Numéraux & 1090 & 604 & 1694 \\
\hline Adjectifs-pronoms & 21322 & 11140 & 32462 \\
\hline Verbes & 30832 & 17646 & 48478 \\
\hline Adverbes & 10399 & 6007 & 16406 \\
\hline Prépositions & 10008 & 5614 & 15622 \\
\hline Conj. de coord. & 12482 & 5564 & 18046 \\
\hline Conj. de subord. & 3501 & 2392 & 5893 \\
\hline TOTAL & 134946 & 76337 & 211283 \\
\hline
\end{tabular}

Écarts réduits :

\begin{tabular}{|l|l|l|}
\hline & Corpus de traductions & Corpus de comparaison \\
\hline Substantifs & $-4,97318$ & 6,6122 \\
\hline Adjectifs & $-1,52846$ & 2,0322 \\
\hline Numéraux & 0,24461 & $-0,3252$ \\
\hline Adjectifs-pronoms & 4,08769 & $-5,4349$ \\
\hline Verbes & $-0,74331$ & 0,9883 \\
\hline Adverbes & $-0,77641$ & 1,0323 \\
\hline Prépositions & 0,30296 & $-0,4028$ \\
\hline Conj. de coord. & 8,90525 & $-11,8402$ \\
\hline Conj. de subord. & $-4,28436$ & 5,6964 \\
\hline
\end{tabular}

La catégorie qui distingue le plus significativement les traductions des non traductions est celle des conjonctions de coordination, avec un surplus très important pour les premières et un déficit encore plus grand pour les secondes. À l'inverse et dans une moindre mesure, les conjonctions de subordination apparaissent en déficit dans le corpus de traductions et en surplus dans l'autre. Une différence se marque aussi dans la 
distribution des substantifs, avec un déficit dans le corpus de traductions et un excédent dans le corpus de comparaison. La distribution des adjectifs-pronoms semble liée à celle des substantifs, car, à l'inverse, ils sont employés en surplus dans le corpus de traductions et en déficit dans l'autre. Cette première analyse fait donc apparaître des variations entre les deux corpus, notamment au niveau de la structure phrastique: le groupe des traductions présentant, d'une manière générale, un style beaucoup plus coordonnant que subordonnant, à l'inverse des textes non traduits.

\section{Corpus global}

60 Il convient à présent de s'interroger sur la distribution des catégories entre les textes. Est-ce que les caractéristiques observées dans le sous-corpus de traductions se retrouvent dans toutes les traductions ou bien est-ce que certains textes se distinguent plus que d'autres par ces emplois particuliers ? L'analyse factorielle est un bon moyen d'étudier la distribution des catégories grammaticales, car elle peut prendre en compte simultanément un nombre important de variables et donner à voir de multiples associations entre les textes étudiés et les paramètres retenus comme facteurs d'analyse. Ici elle peut représenter sur le même graphique les catégories et les textes, ce qui n'était pas possible dans le calcul général de la connexion grammaticale étant donné le nombre de paramètres pris en compte. Les principes de lecture du graphique ne permettent normalement pas d'interpréter directement une proximité ou une opposition entre un point représentant une ligne du tableau (ici les catégories) et un point représentant une colonne (les textes) : il faut toujours considérer un point-ligne par rapport à l'ensemble des points-colonnes ou l'inverse, même si dans la pratique cela revient à peu près au même (ÉVRARD, MELLET, 1998 : 140-144).

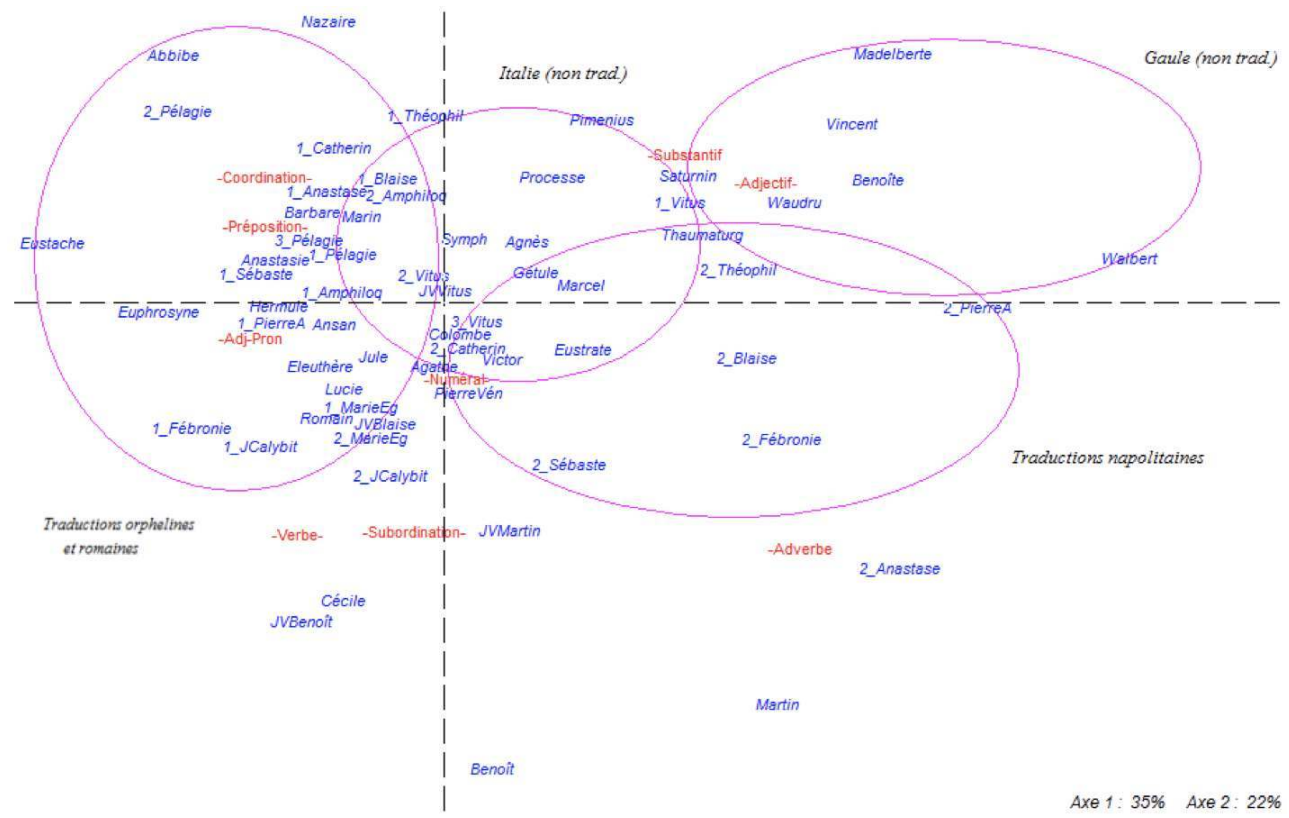

Analyse factorielle de la distribution des catégories grammaticales à travers le corpus global

61 Sur le graphique, on voit une certaine opposition entre les textes non traduits, dans le cadran supérieur droit (même si les textes italiens sont plus centrés que les textes gaulois), du côté des substantifs et des adjectifs, et les traductions (à l'exception des napolitaines), à gauche, du côté des conjonctions de coordination, des adjectifs-pronoms 
et des prépositions; mais on voit aussi que, à l'intérieur des groupes, les textes se répartissent de manière plus ou moins homogène, certains s'écartant plus que d'autres, en raison d'une attirance ou d'une opposition envers une ou l'autre catégorie. Seul le retour aux données chiffrées permet de comprendre leur position. Par exemple, la Vie de Walbert, située à l'extrémité droite, est caractérisée par un suremploi très significatif de substantifs, d'adjectifs et d'adverbes (3 catégories situées à droite par le premier facteur) et par un déficit significatif en conjonctions de coordination et en adjectifs-pronoms (à gauche). La traduction de la Passion d'Eustache, située à l'autre extrémité, présente les caractéristiques inverses. Les Vies de Martin et de Benoît, situées en bas du graphique, montrent un emploi significativement élevé d'adverbes et de conjonctions de subordination (situées en bas par le deuxième facteur), contrebalancé par un déficit en conjonctions de coordination (en haut). La deuxième traduction de la Passion d'Anastase le Perse, à droite, s'explique par un suremploi très significatif d'adverbes.

\section{Sous-corpus italien}

L'analyse suivante ne porte plus que sur le sous-corpus italien et confirme ce qui a été dit. Le premier facteur continue à opposer les traductions napolitaines, qu'il situe du côté des adverbes et des adjectifs, aux autres traductions, qu'il place du côté des conjonctions de coordination et des prépositions. Il corrobore aussi la spécificité de la deuxième traduction de la Passion d'Anastase, à droite, et de la Passion d'Eustache, à gauche. Le deuxième facteur s'attache surtout aux substantifs, qu'il situe en bas et qu'il oppose aux adjectifs-pronoms (en haut); dans une moindre mesure, il marque une opposition entre les conjonctions de coordination et celles de subordination. Il permet de regrouper presque tous les textes non traduits sous l'axe horizontal. Il contribue aussi à souligner l'originalité de la deuxième traduction de la Passion d'Anastase et celle de la Vie amalfitaine de Jean Calybite, qu'il situe en haut, du côté des adverbes, ainsi que celle de la Vie de Grégoire le Thaumaturge, qu'il situe tout en bas, près des substantifs.

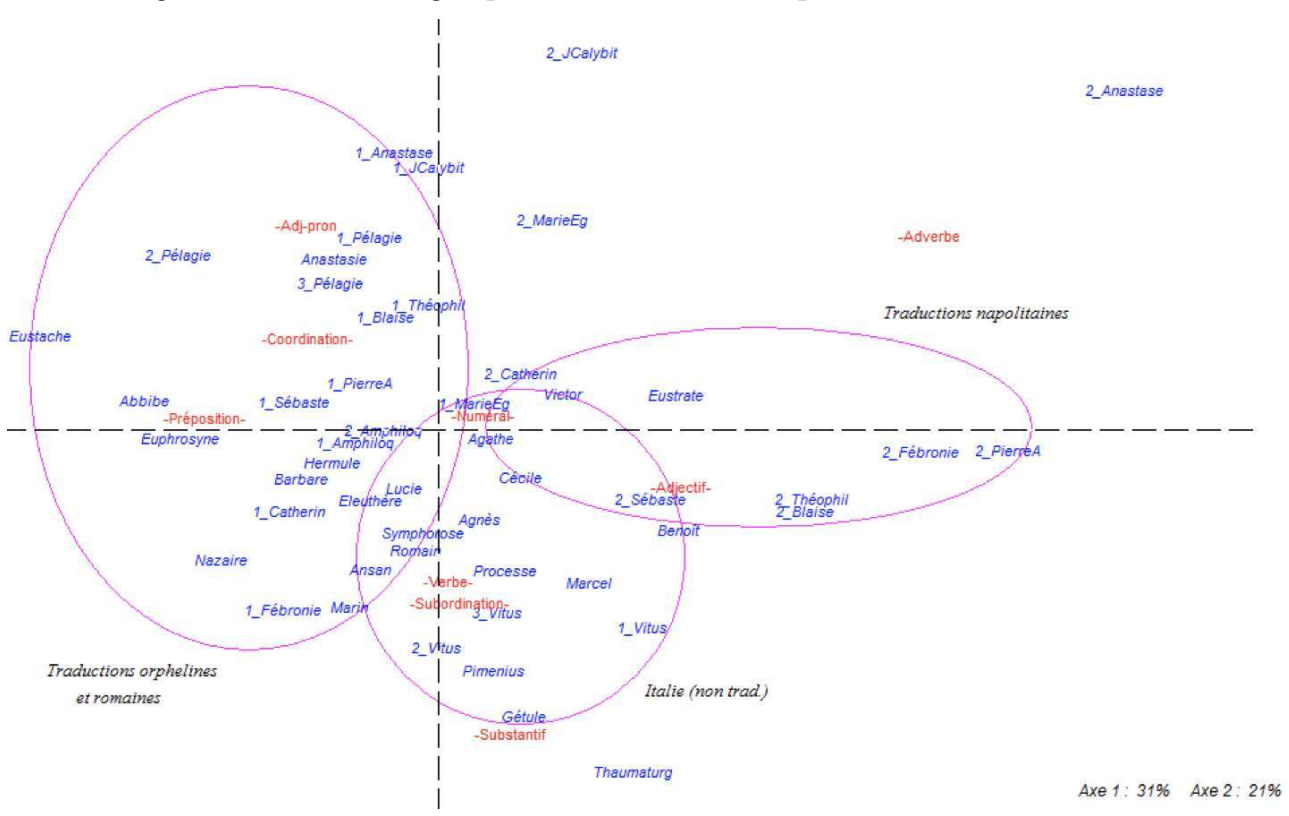

Analyse factorielle de la distribution des catégories grammaticales à travers le sous-corpus italien 


\section{Représentation en histogrammes de la distribution des catégories} textes et les catégories, en illustrant, au moyen de bâtons, la distribution de chacune des catégories à travers l'ensemble du corpus. Elle fait apparaître clairement ce qui caractérise chaque texte du point de vue de la distribution des catégories grammaticales et la manière dont chaque catégorie est distribuée dans les textes et donc dans les groupes. Les catégories sont en effet rarement distribuées de façon régulière à travers un corpus, et des écarts se laissent observer entre les effectifs réels et les effectifs théoriques que l'on s'attendrait à avoir dans l'hypothèse d'une répartition homogène, faite en fonction de la longueur des textes. La signification de ces écarts est mesurée ici par un calcul de l'écart réduit, qui prend en compte la taille des textes et celle du corpus. Sur les histogrammes, les textes sont répartis de part et d'autre d'une ligne médiane, qui représente la valeur zéro de l'écart réduit. Les surplus sont représentés par les bâtons rouges qui se trouvent au-dessus de la ligne et les déficits par les bâtons bleus qui sont en dessous. Les lignes pointillées symbolisent le seuil au-delà duquel l'écart est significatif (en surplus ou en déficit) et en deçà duquel il ne l'est pas. Dans les faits, un surplus d'une catégorie dans un texte ne signifie pas que ce texte utilise plus cette catégorie qu'un autre texte ou qu'il emploie plus cette catégorie qu'une autre ; cela veut dire qu'il l'utilise plus, proportionnellement à sa taille, que ce que laisserait attendre une distribution homogène au sein du corpus. De même, un déficit désigne un emploi proportionnellement moins important que ce que l'on était en droit d'attendre.

Sur les histogrammes, les 15 premiers bâtons représentent les textes italiens qui ne sont pas des traductions, viennent ensuite les traductions appelées orphelines (18 au milieu), puis les traductions dûment localisées à Rome (6), les traductions napolitaines (9) et les traductions amalfitaines (2 derniers). Même si tous les emplois ne sont pas significatifs, le premier histogramme montre que la distribution des substantifs est un des facteurs distinctifs entre les textes italiens non traduits et la majorité des traductions, mises à part les napolitaines. À Naples, on voit que le surplus dans la Vie de Grégoire le Thaumaturge est proportionnellement très élevé, ce qui explique sa position dans l'analyse factorielle.

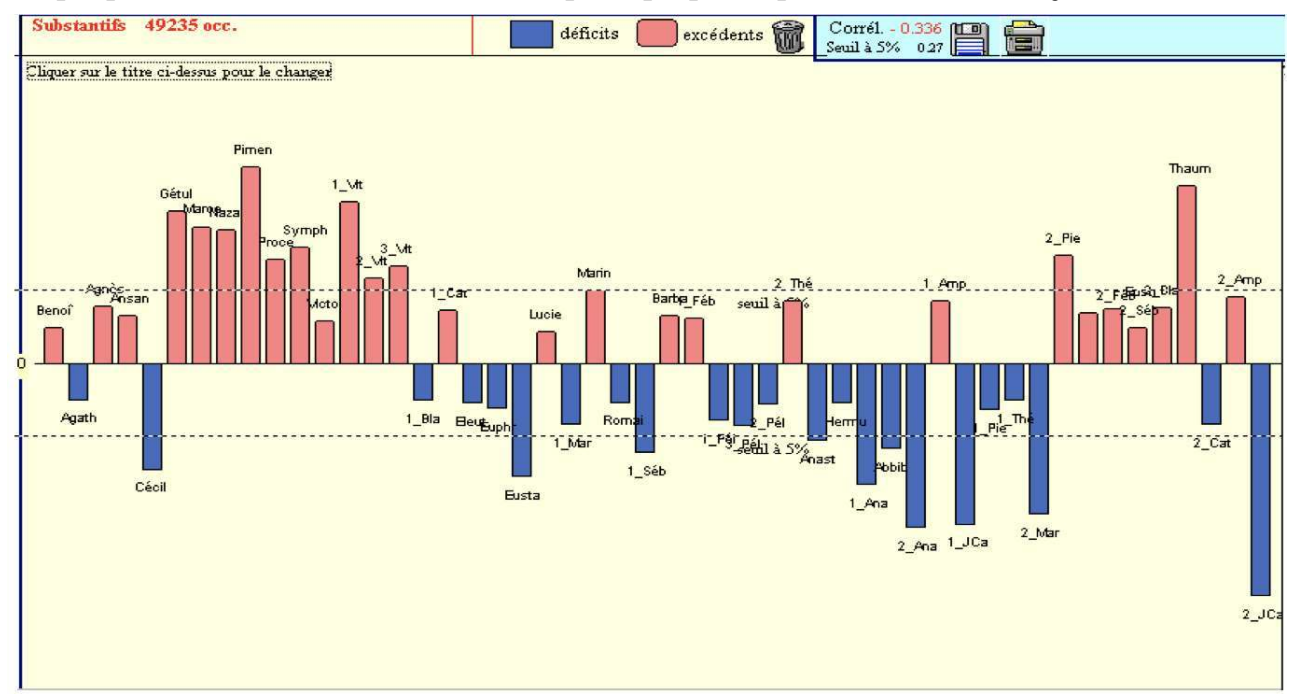

Distribution des substantifs dans le sous-corpus italien 
L'histogramme suivant compare la distribution des substantifs à celle des adjectifs-pronoms. Ici, les bâtons bleus représentent les substantifs et les rouges les adjectifs. Les surplus se trouvent au-dessus de l'axe et les déficits en dessous. Il y a donc pour chaque texte deux bâtons. L'emploi des adjectifs-pronoms apparait souvent opposé à celui des substantifs, comme on avait pu le voir sur le graphique de l'analyse factorielle, même si, comme son nom l'indique, cette catégorie regroupe tous les adjectifs-pronoms, qu'ils soient employés comme pronoms ou comme adjectifs. On note d'ailleurs un taux de corrélation inverse très significatif $(a ̀-0,634)$ entre les courbes de distribution des deux catégories ${ }^{14}$.

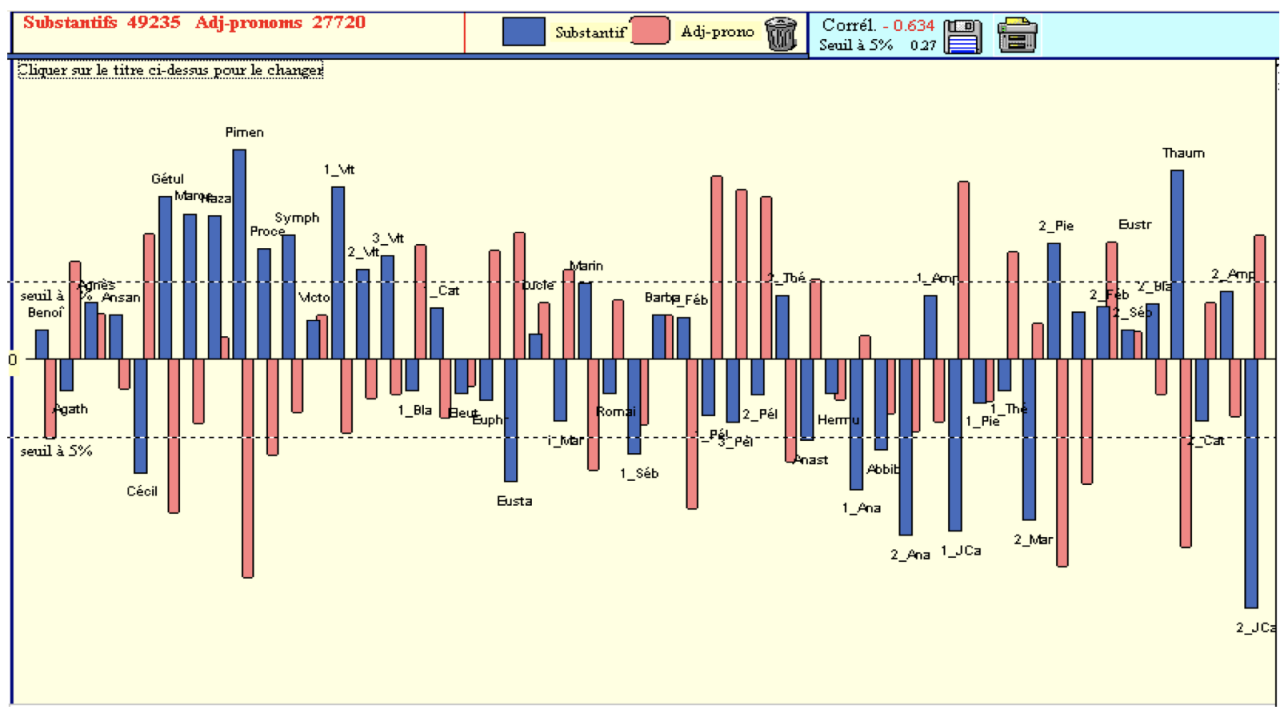

Comparaison des distributions des substantifs (bleu) et des adjectifs-pronoms (rouge) dans le souscorpus italien

L'histogramme qui représente la répartition des conjonctions de coordination est très intéressant. Les surplus commencent avec les premières traductions du corpus (à part 2 cas) et concernent presque toutes les traductions, à l'exception des traductions napolitaines (sauf les deux textes de Paul diacre, qui sont caractérisés par un surplus). Le déficit de la deuxième traduction de la Passion d'Anastase ressemble à ceux de Naples.

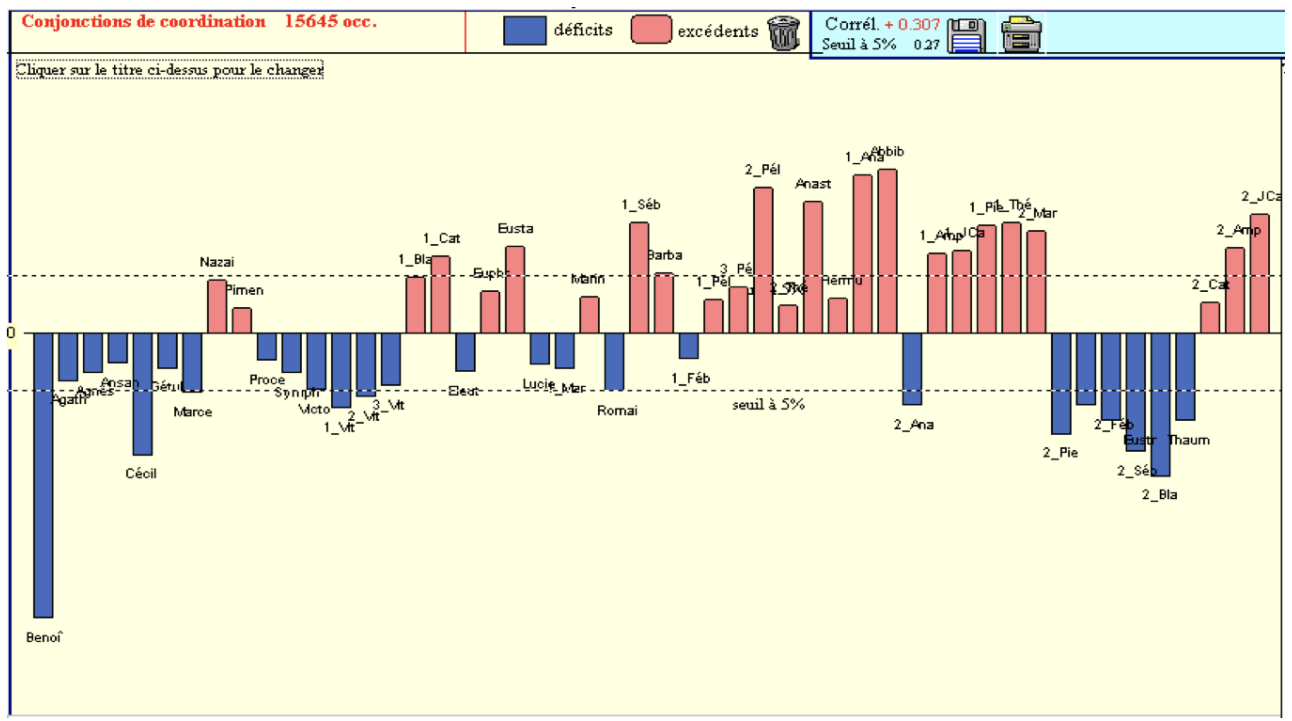

Distribution des conjonctions de coordination dans le sous-corpus italien 
67 seuil de signification, on peut voir qu'il y a plus de déficits que de surplus dans les
traductions, mais que certains surplus sont tout de même significatifs. Le groupe
napolitain est divisé en deux, tout comme celui des textes non traduits. Cette distribution
ne permet donc pas d'opposer les traductions aux textes non traduits aussi nettement que
la distribution des conjonctions de coordination. La comparaison des deux courbes
montre qu'à un surplus en coordination correspond souvent un déficit en subordination
(le taux de corrélation inverse est d'ailleurs assez significatif: $-0,554)$, mais quelques
textes montrent un déficit ou un surplus des deux ${ }^{15}$.

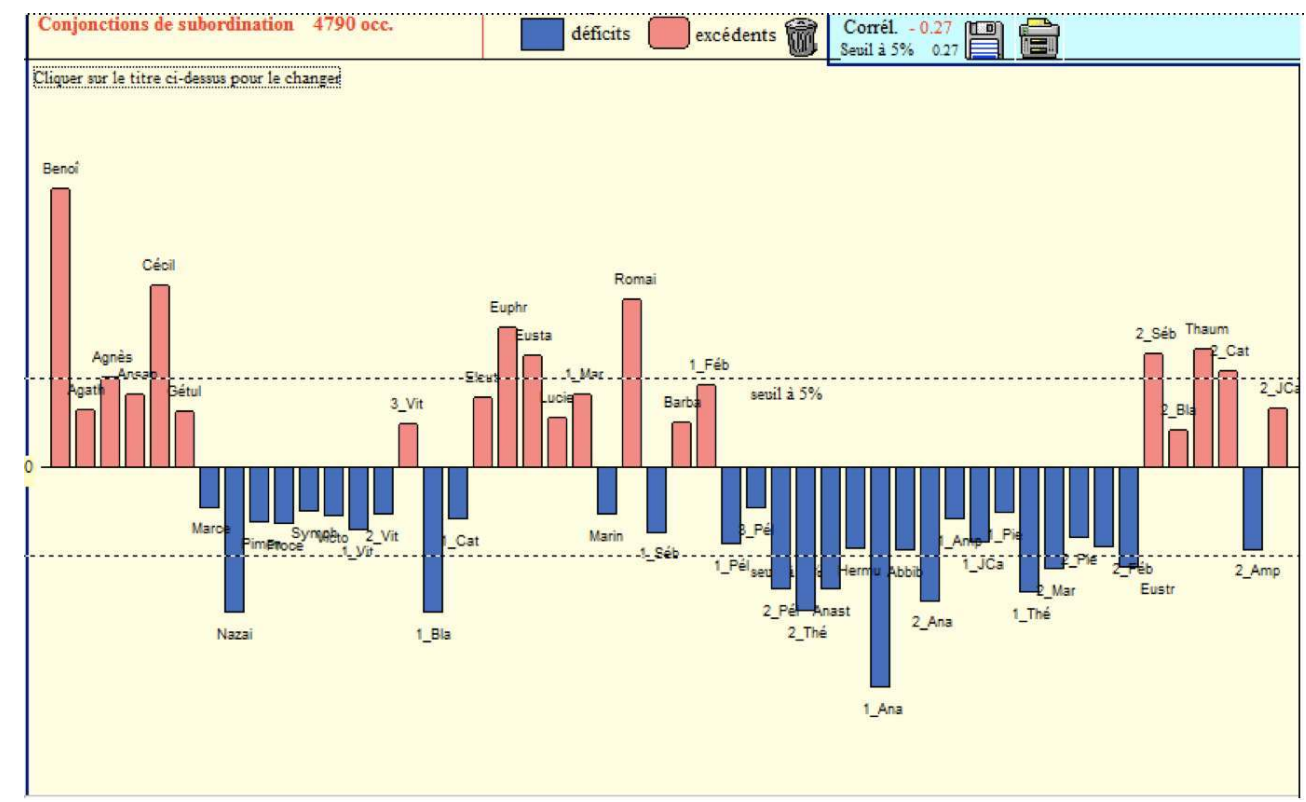

Distribution des conjonctions de subordination dans le sous-corpus italien

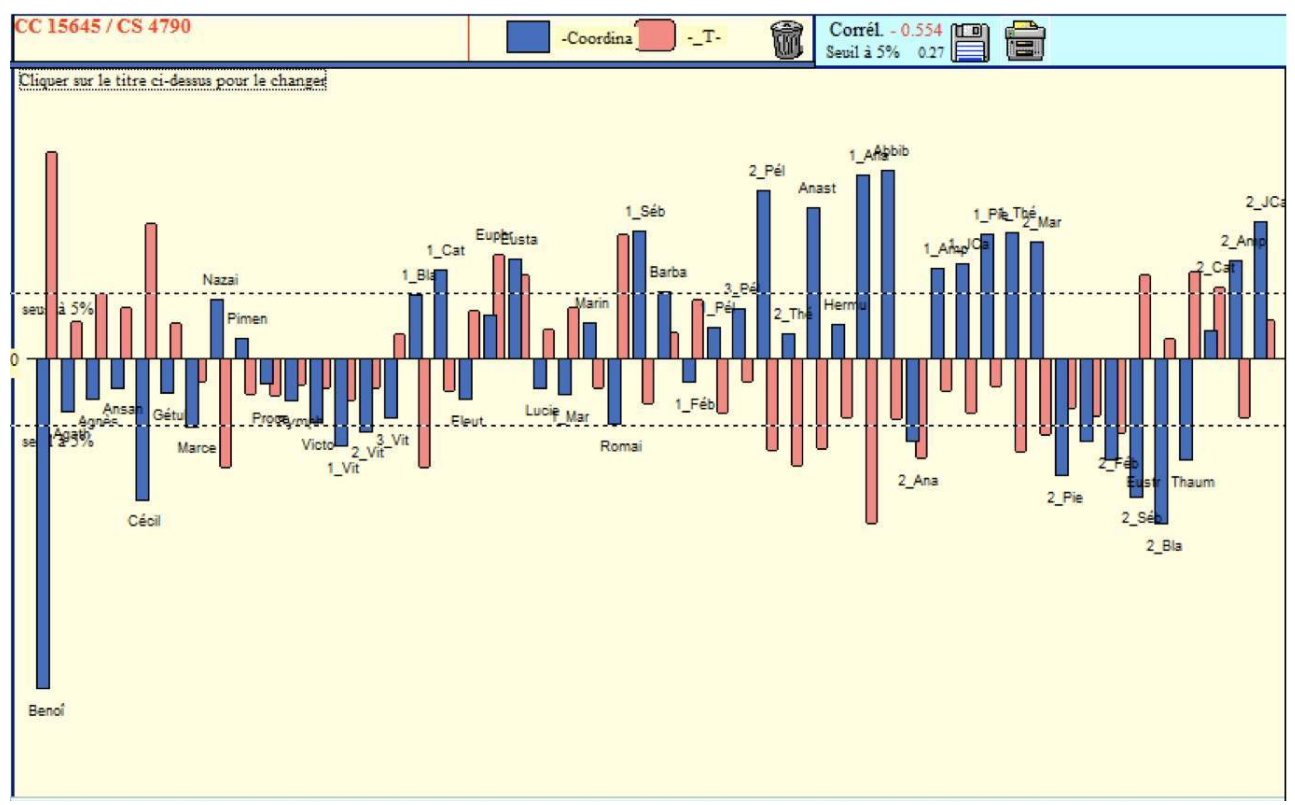

Comparaison des distributions des conjonctions de coordination (bleu) et des conjonctions de subordination (rouge) dans le sous-corpus italien 
Les deux histogrammes suivants montrent que les traductions napolitaines sont bien caractérisées par un emploi significativement élevé d'adjectifs et d'adverbes, qui les distinguent nettement des autres traductions, et même des textes non traduits en ce qui concerne les adverbes. On retrouve un suremploi d'adjectifs très significatif dans l'Histoire de Théophile et dans la deuxième traduction de la Passion d'Anastase, qui est aussi caractérisée par le plus grand surplus d'adverbes.

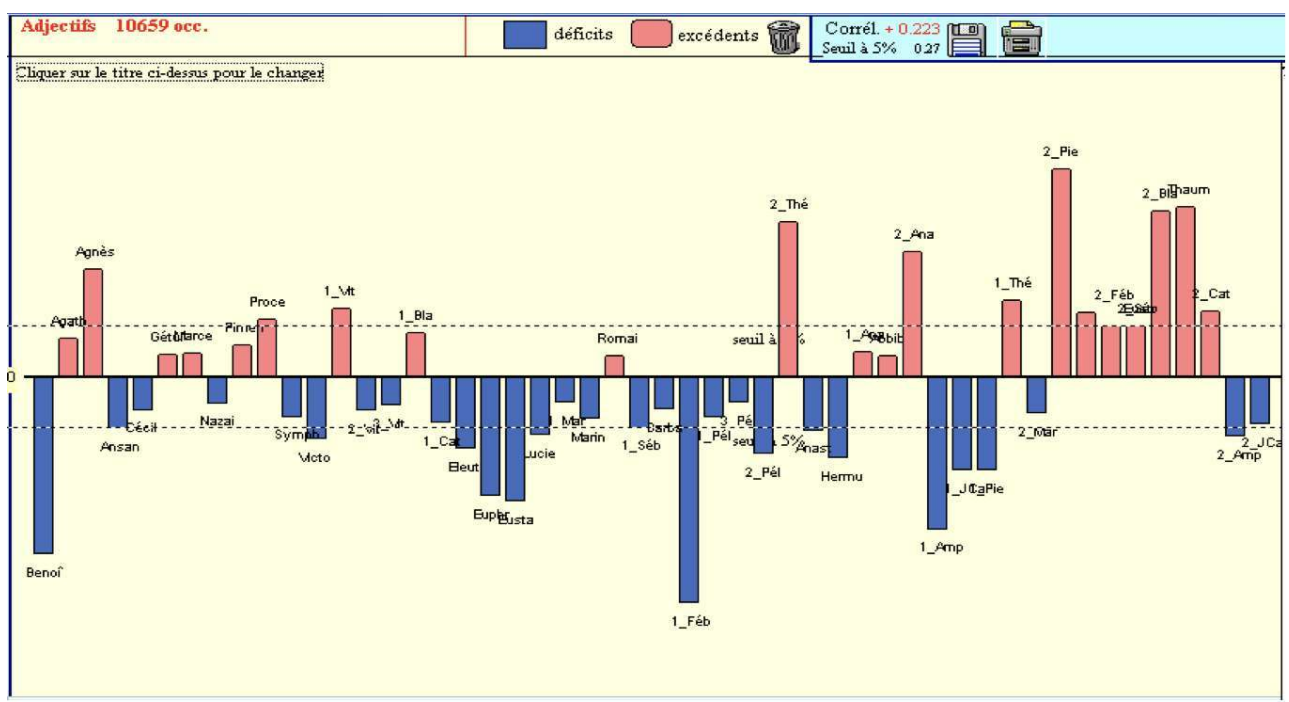

Distribution des adjectifs dans le sous-corpus italien

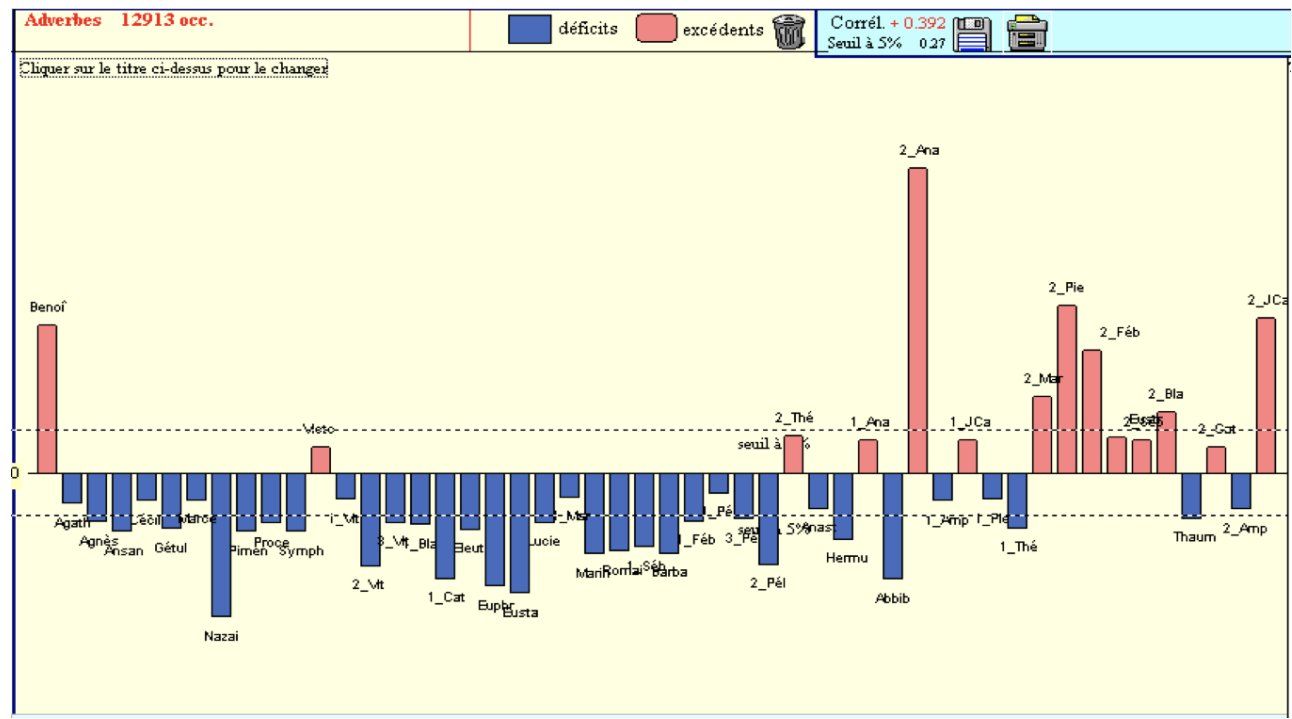

Distribution des adverbes dans le sous-corpus italien

69 La distribution des verbes ne permet pas d'opposer un groupe à un autre. Les bâtons se répartissent en surplus et en déficits dans tous les groupes, dépassant peu le seuil de signification. Certains emplois sont toutefois très significatifs, comme le surplus dans la Passion de Cécile et dans la Passion de Fébronie, ou le déficit dans la traduction napolitaine de la Passion de Pierre d'Alexandrie. 


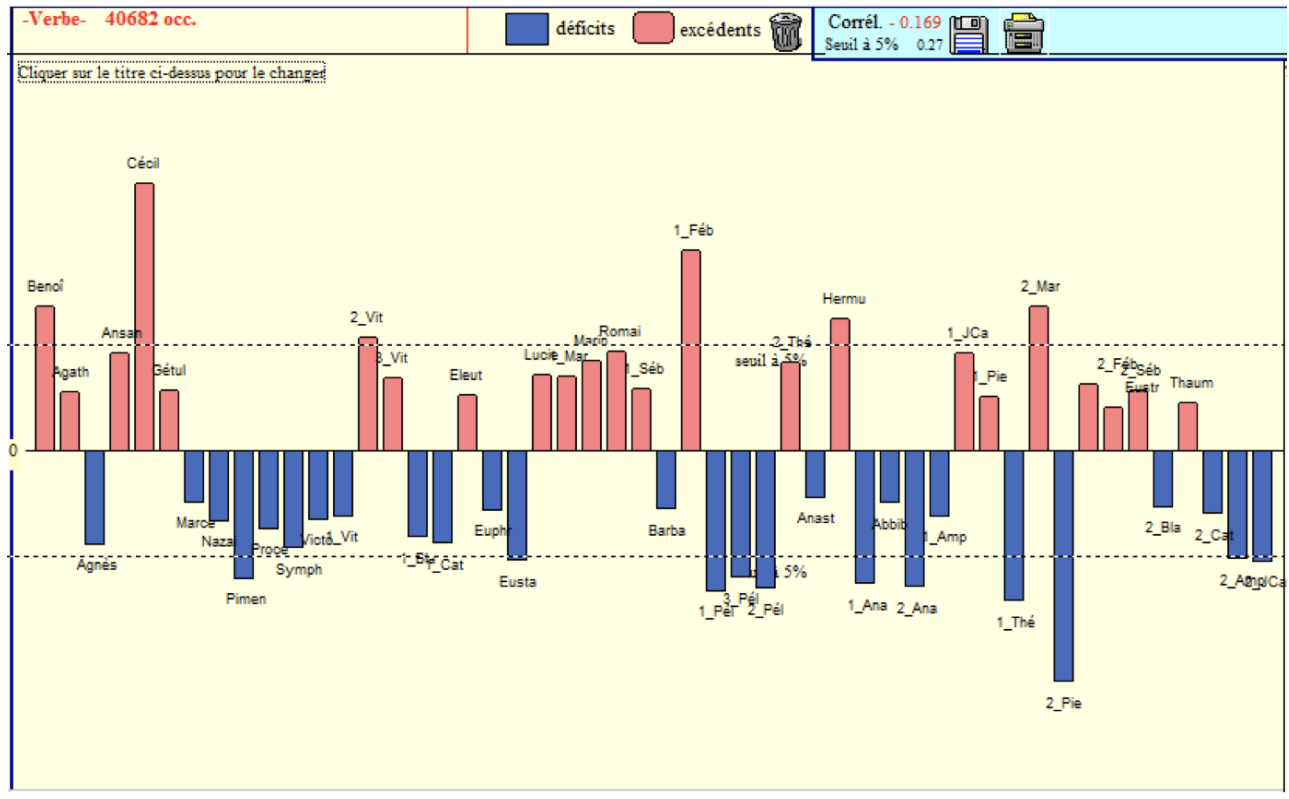

Distribution des verbes dans le sous-corpus italien

Quant aux prépositions, la courbe de distribution semble indiquer qu'elles sont plutôt en surplus dans les traductions, avec quelques surplus très significatifs (Passion d'Eustache, d'Abbibe et première traduction de la Passion d'Anastase), qui les opposent aux traductions de Naples, majoritairement caractérisées par un déficit (tout comme l'Histoire de Théophile et la deuxième Passion d'Anastase). Les textes non traduits montrent, pour leur part, autant de déficits que de surplus.

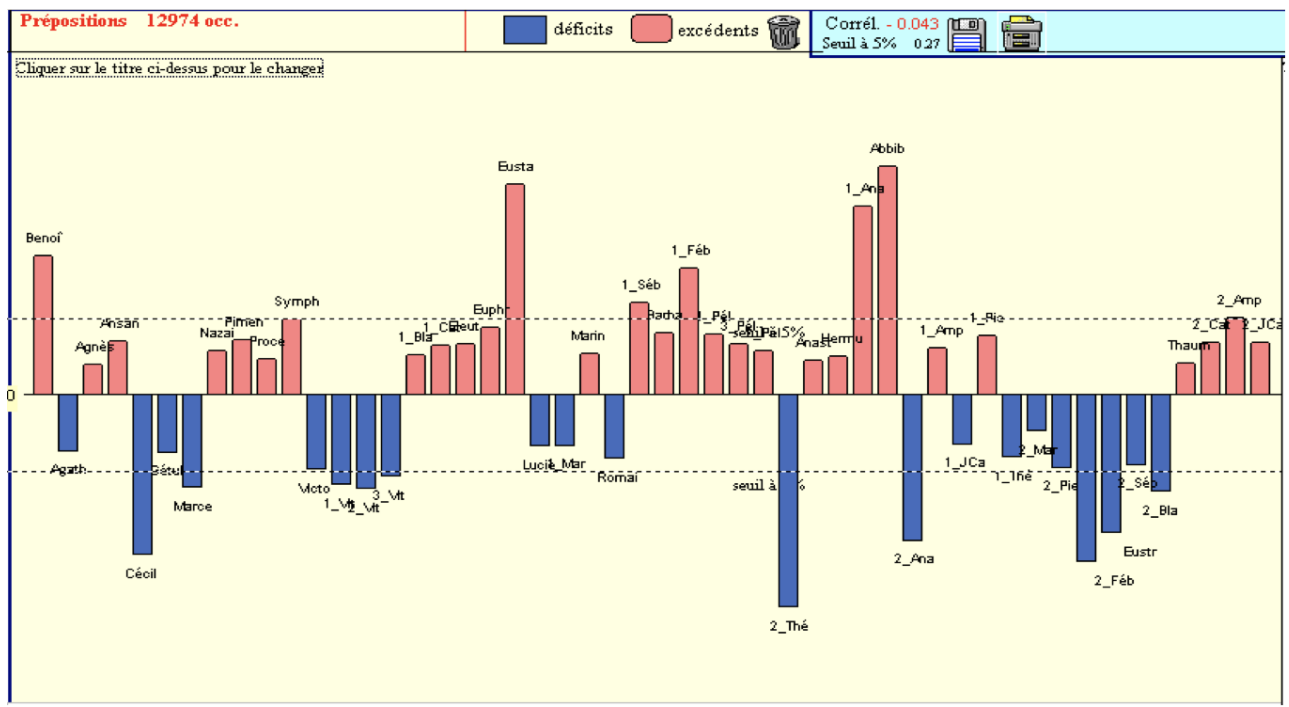

Distribution des prépositions dans le sous-corpus italien

\section{Caractérisation des groupes}

71 L'analyse de la distribution des catégories grammaticales permet donc de préciser en quoi et dans quelle mesure les traductions se distinguent des textes non traduits. Elle permet aussi de caractériser les différents groupes et de repérer les emplois significatifs. Plus précisément, elle permet de tracer le profil d'un texte en fonction de sa manière d'utiliser les différentes catégories par rapport au reste du corpus. 


\section{Traductions / non traductions}

coordination qui serait l'élément le plus distinctif entre les traductions et les non traductions. Le grec utilise naturellement beaucoup plus de particules que le latin. Les traduire au moyen de conjonctions est un procédé courant chez les traducteurs. Il n'est donc pas surprenant de trouver une surabondance de conjonctions de coordination dans les traductions du grec ; la voici confirmée par les chiffres et les graphiques. Toutefois, si la profusion de ces conjonctions peut s'expliquer par le poids du modèle grec et se révéler caractéristique des traductions, elle n'en reste pas moins dépendante du style et du niveau des traducteurs. En effet, elle concerne avant tout les traductions littérales, et surtout celles qui ont été faites au mot-à-mot, qui cherchent un équivalent latin pour chaque mot grec. Les versions napolitaines, traduites plus librement et rédigées dans un style plus soigné et plus recherché, se démarquent de façon très significative sur ce point. Un examen plus approfondi de leur distribution (qu'il n'est pas possible de présenter ici) révèle des divergences encore plus profondes (comme le surplus napolitain en explicatives alors que les autres groupes sont caractérisés par un surplus en copulatives et en adversatives, ou bien son déficit en et, employé en surplus ailleurs et contrebalancé à Naples par un suremploi en que). Les conjonctions appartiennent au groupe des motsoutils, indispensables à la langue, mais qui relèvent le plus souvent de l'inconscient de l'auteur. À ce titre, elles peuvent trahir des tics de langage. Certains auteurs accordent aussi plus de soin à l'organisation de leur discours et à la variation du vocabulaire. Les conjonctions de coordination constituent donc un élément linguistique qui peut se montrer révélateur du niveau ou du style d'un auteur, en même temps qu'il peut nous renseigner sur la qualité de sa traduction.

73 La distribution des substantifs semble aussi distinguer les textes non traduits par des surplus et les traductions par des déficits. Ce phénomène est toutefois plus délicat à interpréter. Certains des déficits relevés dans les traductions semblent s'expliquer par un emploi important en adjectifs-pronoms, qui est aussi révélateur d'une manière d'écrire. Les pronoms peuvent en effet trahir un relâchement dans l'écriture, par exemple par abus d'anaphore à longue portée ou par indéfinition du référent, alors que le fait de nommer ou dénommer implique plus de précision dans l'écriture et montre le désir de bien cerner le référent. D'autres déficits, mais apparemment moins nombreux, sont contrebalancés par un emploi plus important en verbes, ce qui suggère un style plus axé sur l'action, sur la succession des faits, un style peut-être moins intellectualisant ou moins conceptualisant que celui qui regorge de substantifs.

\section{Traductions napolitaines}

74 Leur originalité a été soulignée à plusieurs reprises, tant sur le plan lexical que syntaxique. L'emploi des catégories grammaticales met en évidence un niveau d'écriture plus élevé. Les surplus observés dans la distribution des adjectifs et des adverbes suggèrent en effet un style développé et imagé et le surplus en substantifs en souligne le côté intellectuel. Le déficit en conjonctions de coordination et le surplus en conjonctions de subordination, relevé dans certains textes en tout cas, confirment une manière d'écrire construite et structurée. Le déficit en prépositions renforce l'idée de textes bien écrits, dans lesquels la flexion nominale joue encore un rôle suffisamment important 
pour ne pas imposer un recours trop fréquent aux prépositions. Ces observations restent, à ce stade, assez générales, mais elles peuvent être précisées pour chaque texte. En tout cas, elles correspondent à ce qui est généralement dit des traductions napolitaines, destinées, pour la plupart, à remplacer des versions anciennes maladroites et trop littérales.

75 Selon Paolo CHIESA (1989-1990 : 71-73), l'école de Naples aurait connu une évolution dans son mode de traduction, qui peut être définie en trois phases, allant vers une liberté de plus en plus grande vis-à-vis du modèle de départ. La première phase coïncide avec l'épiscopat d'Athanase II et concerne les traductions de Paul diacre et de Guarimpotus, caractérisées par l'emploi d'une méthode encore assez traditionnelle. La deuxième est représentée par Jean diacre, à la fin du IX ${ }^{\mathrm{e}}$ et au début du $\mathrm{X}^{\mathrm{e}}$ siècle, qui prend un peu plus de liberté vis-à-vis de son modèle ; la finalité pastorale y est de plus en plus évidente. La troisième, dominée par les figures des sous-diacres Pierre et Bonitus au milieu du $\mathrm{X}^{\mathrm{e}}$ siècle, est caractérisée par des versions beaucoup plus libres et par l'insertion de vers à l'intérieur du texte en prose. Le particularisme des textes de Paul diacre semble s'expliquer par cette évolution. Les calculs de la distance grammaticale avaient montré que ses deux textes étaient éloignés du reste de Naples et plus proches des traductions orphelines et romaines. L'analyse de la distribution des catégories en explique les raisons. Ce qui les caractérise le plus, c'est l'emploi des conjonctions et la préférence donnée à la coordination plutôt qu'à la subordination. La Vie de Marie se distingue aussi par son déficit en substantifs et en adjectifs (même si ce dernier n'est pas significatif) et la Pénitence de Théophile par son déficit en adverbes, des catégories qui sont en surplus dans les autres traductions napolitaines.

\section{Traductions romaines}

76 Celles-ci se sont généralement montrées plus proches des traductions orphelines que des napolitaines, aussi bien dans le calcul de la distance lexicale et grammaticale que dans celui de la distribution des catégories. Elles sont avant tout caractérisées par un surplus en conjonctions de coordination, contrebalancé par un déficit en conjonctions de subordination. Leur déficit en substantifs et en adjectifs, ainsi que leur surplus en prépositions contribuent aussi à les rapprocher des traductions orphelines, en les distinguant des napolitaines.

77 Cependant, il faut se rappeler que, dans sa composition, le groupe romain est moins homogène que le groupe napolitain. Il contient trois œuvres d'Anastase le Bibliothécaire, traduites de manière littérale, comme les traductions orphelines, mais rédigées dans un latin plus correct et plus intelligible. Il compte ensuite deux traductions très littérales, de mauvaise qualité - la Passion d'Abbibe, par Léo, et la Passion d'Anastase le Perse, par Thédore de Tarse - ainsi qu'une deuxième traduction de la Passion d'Anastase, signée par un certain Grégoire. Celui-ci était traditionnellement rattaché à l'école napolitaine, mais il a été relocalisé à Rome par l'éditrice (VIRCILLO FRANKLIN, 2004 : 96-100). La première traduction de la Passion d'Anastase a été faite ad verbum, avec beaucoup de maladresses et d'incorrections, et, comme la Passion d'Abbibe, elle montre beaucoup de points communs avec les traductions orphelines. La deuxième, destinée à remplacer une version ancienne de mauvaise qualité, a été réalisée dans un style plus soigné et plus sophistiqué. À son sujet, il est remarquable que toutes les analyses ont montré qu'elle avait plus de points communs avec les traductions napolitaines qu'avec celles d'Anastase le Bibliothécaire. 
Cela ne condamne pas l'hypothèse avancée par l'éditrice, mais cela ajoute un élément, d'ordre philologique, en faveur de la théorie traditionnelle.

\section{Traductions orphelines}

78 Cette appellation est, comme cela a déjà été dit, un peu grossière, puisque, faute de mieux, elle réunit les traductions qui ne sont pas connues comme étant de Naples, ni de la Rome des IX- $\mathrm{X}^{\mathrm{e}}$ siècles. Ce sont des traductions anonymes, généralement plus anciennes, dont plusieurs ont sans doute été rédigées à Rome, certaines au Nord, d'autres au Sud. Ce groupe contient aussi les deux réécritures, l'Histoire de Théophile et la Passion de Pélagie, parce qu'elles ont vraisemblablement été composées dans le Nord de l'Italie, comme la Passion de Barbare, de Fébronie et l'autre traduction de Pélagie.

79 Les traductions orphelines ont généralement été réalisées ad verbum. Ces traductions littérales sont souvent calquées sur le grec, ce qui peut expliquer les surplus en conjonctions de coordination et sans doute aussi en prépositions. Ce sont aussi le plus souvent des textes simples, écrits sans grande recherche stylistique. L'importance est donnée au contenu et non à la forme, ce qui peut également expliquer les déficits observés en adjectifs et en adverbes. L'opposition entre les traductions napolitaines et les traductions orphelines correspond bien à deux manières de traduire très différentes. Les histogrammes ont toutefois montré que, même si on pouvait en titrer des tendances générales, il n'y avait pas homogénéité parfaite dans le groupe. Les déficits en adjectifs et en adverbes sont les caractéristiques les plus homogènes du groupe, qui les opposent clairement aux textes napolitains. Les autres catégories sont distribuées de façons plus variées à l'intérieur du groupe, avec des surplus et des déficits plus ou moins significatifs. Cela vaut aussi pour les conjonctions de coordination, même s'il y a plus de surplus significatifs que de déficits significatifs.

80 L'Histoire de Théophile a montré plus de points communs avec les traductions napolitaines. C'est un remaniement assez profond de la Poenitentia Theophili de Paul diacre, rédigé dans un style recherché et plus élevé (CHIESA, 1994), ce qui explique sa proximité avec les textes de Naples.

\section{Conclusion}

81 L'analyse quantitative fournit donc des éléments nouveaux à l'étude de la traduction hagiographique médiolatine. Elle permet d'étudier un large corpus de traductions et de le confronter à un corpus de textes non traduits, afin d'identifier ce qui les distingue sur le plan linguistique. En comparant les traductions les unes aux autres, elle mène en outre à leur classification et à l'identification des caractéristiques linguistiques des différents groupes et des différentes traductions prises individuellement. L'objectif de ce travail n'était pas de caractériser tous les textes, mais plutôt de montrer l'intérêt de recourir à ces méthodes.

Pour mon propos, je suis partie des analyses les plus générales mesurant la distance intertextuelle pour aller vers des analyses plus spécifiques, comme celle des catégories grammaticales. Des analyses plus fines ont été effectuées dans le cadre de ma thèse, où les résultats présentés sont plus précis, mais les exposer ici nécessiterait de plus amples développements et tel n'était pas l'objectif. L'étude des conjonctions de coordination est très intéressante, de même que celle des participes et des propositions complétives 
introduites par quod ou quia comparées aux propositions infinitives. Ces trois phénomènes linguistiques sont connus comme pouvant être une trace de l'influence du grec : l'analyse quantitative permet de les mesurer.

Une approche telle que celle-ci, basée sur une analyse comparative des traductions, sur des données chiffrées et objectives, contribue donc à améliorer la connaissance que nous pouvons avoir de la traduction hagiographique médiolatine, un domaine dans lequel beaucoup reste à faire. Des recherches en cours, notamment avec Monique Goullet, et l'ajout de nouveaux textes au corpus permettront d'avancer un peu plus dans cette direction.

\section{BIBLIOGRAPHIE}

BARDY G., La question des langues dans l'Église ancienne, Paris, Beauchesne, 1948 (Études de théologie historique).

BARTHELEMY J.-P. - X. LUONG, « Représenter les données textuelles par les arbres...», dans S. MELLET

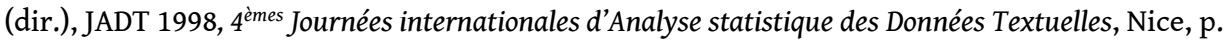
49-71.

BARTHELEMY J.-P. - X. LUONG - S. MELLET, « Prenons nos distances pour comparer des textes, les analyser et les représenter », dans X. LUONG (dir.), La distance intertextuelle, Corpus 2 (2003), p. 5-18. BERSCHIN W., Griechisch-lateinisches Mittelalter. Von Hieronymus zu Nikolaus von Kues, Bern-Munich, Francke, 1980. Traduction italienne par E. LIVREA, Medioevo greco-latino. Da Gerolamo a Niccolò Cusano, Naples, Liguori, 1989 (Nuovo Medioevo, 33).

BERSCHIN W., « Les traducteurs d'Amalfi au XIe siècle », dans G. CONTAMINE (dir.), Traduction et traducteurs au Moyen Âge. Actes du colloque international du CNRS, organisé à Paris, IRHT, 26-28 mai 1986 , Paris, Éditions du CNRS, 1989 (Documents, études et répertoires), p. 163-168.

BRUNET É., « Peut-on mesurer la distance entre deux textes ? », dans X. LUONG (dir.), La distance intertextuelle, Corpus, 2 (2003), p. 47-70.

CHIESA P., « Ad verbum o ad sensum ? Modelli e coscienza metodologica della traduzione tra tarda antichità e alto medioevo ", Medioevo e Rinascimento, I (1987a), p. 1-51.

CHIESA P., « Una traduzione inedita di Anastasio Bibliotecario ? Le « vitae » latine di sant'Anfilochio », Studi Medievali, 28 (1987b), p. 879-903.

CHIESA P., «Traduzioni e traduttori dal greco nel IX secolo : sviluppi di una tecnica », dans Giovanni Scoto nel suo tempo. L'organizzazione del sapere in età carolingia. Atti del XXIV Convegno storico internazionale (Todi, 11-14 ottobre 1987), Spolète, CISAM, 1989 (Atti dei Convegni dell'Academia Tudertina e del centro di Studi sulla Spiritualità medievale, n. s., 1), p. 171-200.

CHIESA P., « Le traduzioni dal greco : l'evoluzione della scuola napoletana nel X secolo », Mittellateinisches Jahrbuch, 24-25 (1989-1990), p. 67-86.

CHIESA P., Le versioni latine della Passio Sanctae Febroniae. Storia, metodo, modelli di due traduzioni agiografiche altomedievali, Spolète, CISAM, 1990 (Bibliotheca di Medioevo Latino, 2). 
CHIESA P., «L'Historia Theophili Atheniensis : il più antico rifacimento latino della Poenitentia Theophili », Aevum, 68 (1994), 2, p. 259-281.

CHIESA P., « Le traduzioni », dans G. CAVALlo (dir.), Lo Spazio letterario del medioevo, I/3, Rome, Salerno, 1995, p. 165-196.

CHIESA P., « Traduzioni e traduttori a Roma nell'alto medioevo », dans Roma fra Oriente e Occidente, 2 vol., Spolète, CISAM, 2002 (Settimane di studio del Centro italiano di studi sull'alto medioevo, 49 ), p. 455-492.

D'ANGELO E., Pietro Subdiacono napoletano. L'opera agiografica, Florence, Sismel, 2002 (Edizione nazionale dei testi mediolatini, 7, serie I, 4).

D'ANGELO E., « Agiografia latina del Mezzogiorno continentale d'Italia (750-1000) », dans G. PHILIPPART (dir.), Hagiographies. Histoire internationale de la littérature hagiographique latine et vernaculaire en Occident des origines à 1550, IV, Turnhout, Brepols, 2007, p. 41-134.

DELEHAYE H., Étude sur le légendier romain, Bruxelles, Société des Bollandistes, 1936 (Subsidia hagiographica, 23).

DEVOS P., « L'œuvre de Guarimpotus, hagiographe napolitain », Analecta Bollandiana, 76 (1958), p. 151-187.

DOLBEAU F., «La vie latine de S. Euthyme : une traduction inédite de Jean diacre napolitain », Mélanges de l'École Française de Rome. Moyen Âge - Temps modernes, 94 (1982), p. 315-335.

DOLBEAU F., « Le rôle des interprètes dans les traductions hagiographiques d'Italie du Sud », dans G. CONTAMINE (dir.), Traduction et traducteurs au Moyen Âge. Actes du colloque international du CNRS, organisé à Paris, IRHT, 26-28 mai 1986, Paris, Éditions du CNRS, 1989 (Documents, études et répertoires), p. 145-162.

DOLBEAU F., «Critique d'attribution, critique d'authenticité. Réflexions préliminaires », Filologia mediolatina, VI-VII (1999-2000), p. 33-61.

ÉVRARD É., «Le laboratoire d'analyse statistique des langues anciennes de l'université de Liège ", Mouvement scientifique en Belgique, 9 (1962), p. 163-169.

ÉVRARD É. - A. BODSON, « Le programme d'analyse automatique du latin », Revue de l'organisation internationale pour l'étude des langues anciennes par ordinateur, 2 (1966), p. 17-46.

ÉVRARD É. - S. MELLET, « Méthodes quantitatives en langues anciennes », Lalies, 18 (1998), p. 111-155. GENETTE G., Seuils, Paris, Éditions du Seuil, 1987.

GOULLET M., Écriture et réécriture hagiographiques. Essai sur les réécritures de Vies de saints dans L'occident latin médiéval (VIII'-XIII s.), Turnhout, Brepols, 2005.

GOULLET M. - C. PHILIPPART DE FOY, « Mesurer les distances entre des textes pré-carolingiens linguistiquement non-normés: le cas de la Passion de sainte Marine ", dans Langues anciennes et analyse statistique : cinquante ans après - Distances textuelles et intertextualités (Rome, Academia Belgica, 19-21 octobre 2011), numéro spécial de la revue Les Études Classiques, à paraître.

GRANIER T., " Naples aux IX ${ }^{\mathrm{e}}$ et $\mathrm{X}^{\mathrm{e}}$ siècles : topographie religieuse et production hagiographique ", dans N. COULET - O. GUYOTJEANNIN (dir.), La ville au Moyen Âge : actes du 120e congrès national des sociétés historiques et scientifiques, 1995, Aix-en-Provence, I, Paris, CTHS, 1999 (format, 35), p. 113-131. 
GRANIER T., «Transformations de l'Église et écriture hagiographique à Naples autour de l'An Mil », dans C. CARozZI - H. TAViANi-CARozZi (dir.), Année Mille An Mil, Aix-en-Provence, Publications de l'Université de Provence, 2002, p. 149-175.

LABBE D. - D. MONIÈRE, « La connexion intertextuelle. Application au discours gouvernemental québécois ", dans M. RAJMAN - J.-C. CHAPPELIER (dir.), JADT 2000, Actes des $5^{\text {èmes }}$ Journées internationales d'Analyse statistique des Données Textuelles, I, Lausanne, EPFL, p. 85-94.

LEONARDI C., «L'agiografia romana nel secolo IX », dans p. RICHE (dir.), Hagiographies, cultures et sociétés. IVe-XII siècles, Actes du colloque organisé à Nanterre-Paris (2-5 mai 1979), Paris, Études augustiniennes, 1981, p. 471-490.

LUONG X., « Analyse arborée des données textuelles : mode d'emploi », Travaux du cercle Linguistique, 16 (1994), p. 25-42.

LUZZATI LAGANÀ F., « Le firme greche nei documenti del Ducato di Napoli », Studi Medievali, 23

(1982), p. 729-752.

PETIMENGin p. (éd.), Pélagie la pénitente. Métamorphoses d'une légende, I. Les textes et leur histoire, Paris, Études augustiniennes, 1981.

PHILIPPART DE FOY C., « La philologie latine aux prises avec les textes hagiographiques », Litterae Hagiologicae, 7-8 (2001), p. 15-31.

PHILIPPART DE FOY C., « Mediolatinitas : légendes hagiographiques et statistique linguistique », Hagiographica, X (2003), p. 37-77.

PHILIPPART DE FOY C., Hagiographie et statistique linguistique : étude d'un corpus de traductions médiolatines d'origine grecque, Thèse de doctorat soutenue à l'Université de Nice en 2008, sous la direction de S. Mellet et de F. Dolbeau (inédite).

SANSTERRE J.-M., Les moines grecs et orientaux à Rome aux époques byzantine et carolingienne (milieu du $V I^{e}$ s. - fin du IX ${ }^{e}$ s.), 2 vol., Bruxelles, Palais des Académies, 1983 (Académie Royale de Belgique, Mémoires de la Classe des Lettres, 2e série, 66, 1).

SIEGMUND A., Die Überlieferung der griechischen christlichen Literatur in der lateinischen Kirche bis zum zwölften Jahrhundert, Munich, Filser, 1949 (Abhandlungen der bayerischen

Benediktinerakademie, 5).

TELFER W., « St. Peter of Alexandria and Arius », Analecta Bollandiana, 67 (1949), p. 17-130.

VIRCILLO FRANKLIN C., The Latin Dossier of Anastasius the Persian. Hagiographic Translations and Transformations, Toronto, Pontifical Institute of Medieval Studies, 2004 (Studies and Texts, 147).

\section{NOTES}

1. Le corpus hagiographique latin compte déjà plus de 13000 textes répertoriés dans la Bibliotheca Hagiographica Latina des Bollandistes.

2. Par exemple, la deuxième Vie de Jean Calybite n'est reprise ni chez Siegmund, ni dans la BHL. La Passio Anastasiae uiduae (BHL 400-401) a d'abord été écrite en latin et n'a pas été traduite en grec avant le début du IX ${ }^{\mathrm{e}}$ siècle (DELEHAYE, 1936 : 245-249).

3. L'anonymat, souvent considéré comme une preuve de la modestie du traducteur, s'expliquerait en réalité, dans bon nombre de cas, par accident ou par suppression des sections signées, telles que le prologue ou l'épilogue, plus fragiles et fréquemment écartées dès que s'estompe le souvenir des personnes mentionnées (DOLBEAU, 1999-2000 : 53-61). On rappellera le 
cas de la Passio Blasii (BHL 1379-1380), qui, après être restée longtemps anonyme, fut attribuée à Guarimpotus par P. DEVos (1958), avant que l'on ne retrouve une version du prologue avec le nom de Bonitus comme auteur.

4. Sur les différents traducteurs, voir aussi DEvos (1958), DOLBEAU (1982), D’ANGELO (2002).

5. Les principes du LASLA ont été décrits à plusieurs reprises (ÉVRARD, 1962 : 163-169; ÉVRARDBODSON, 1966 : 17-46). Pour rappel, l'analyse est faite de manière semi-automatique grâce à un analyseur morphologique, qui propose, pour chaque forme, les différents lemmes et analyses possibles. C'est au philologue que revient le choix final. L'analyse est transcrite sous la forme d'un code alpha-numérique, qui répond à un schéma précis, donnant, en résumé, la catégorie grammaticale et la sous-catégorie, puis le cas et le nombre pour les mots déclinés, la personne, le mode, le temps et la voix pour les verbes. Les propositions principales sont distinguées des subordonnées, lesquelles sont classées par type de subordonnant.

6. Travailler sur les éditions ou sur les manuscrits est une question très délicate, en particulier quand il s'agit d'une étude linguistique. Vu que cette recherche exigeait un large corpus, il n'était pas envisageable d'y ajouter un travail d'édition ni un recours systématique aux manuscrits. L'enjeu de la thèse était avant tout méthodologique et, même s'il faut garder à l'esprit ces limites, celles-ci ne remettent pas en cause les résultats, qui sont basés sur un nombre important d'analyses effectuées sur différents facteurs lexicaux et syntaxiques. Depuis lors, d'autres traductions ont été ajoutées au corpus, comme la Passio Marinae et la Passio Eupraxiae, transmises par le manuscrit Torino BN, D.V.3 (c. 790), et les résultats obtenus avec ces nouveaux textes ne contredisent en rien les résultats précédemment obtenus (GOULLET-PHILIPPART DE FOY, à paraître).

7. Il s'agit du corpus constitué pour le projet Mediolatinitas (PHILIPPART DE FOY 2001 et 2003), qui comprend des textes de Gaule et d'Italie : 1) Vita Martini de Sulpice Sévère, Vita Benedicti de Grégoire le Grand ; 2) Gaule : Passio Benedictae (BHL 1087), Passio Columbae (BHL 1896), Passio Iuliae (BHL 4518b), Vita Madelbertae (BHL 5129), Vita Petri Venerabilis (BHL 6787), Passio Saturnini (BHL 7497), Passio Vincentii (BHL 8621), Vita Walberti (BHL 8775), Vita Waldedrudis (BHL 8776) ; 3) Italie : Passio Agathae (BHL 133), Passio Agnetis (BHL 156), Passio Ansani et Maximae (BHL 515), Passio Caeciliae (BHL 1495), Passio Getulii, Cerealis et soc. (BHL 3524), Passio Marcelli et Apuleii (BHL 5252b), Passio Nazarii et Celsi (BHL 6040), Passio Pimenii (BHL 6849), Passio Processi et Martiniani (BHL 6947), Passio Symphorosae (BHL 7971), Passio Victoris et Coronae (BHL 8559), 3 versions de la Passio Viti (BHL 8711, 8712 et 8713), 4 abbreviationes de Jacques de Voragine (V. Martini, V. Benedicti, P. Blasii et P. m. Sebastenorum).

8. "Anonymes ", « anciennes " ou "non localisées » ne convenaient pas à cause de la présence dans d'autres groupes de textes anonymes, et, dans ce groupe-ci, de textes tardifs ainsi que d'hypothèses de localisation (à ce sujet, j'ai choisi de réserver l'appellation « romaines » aux traductions attachées à un auteur romain plus ou moins bien identifié).

9. Sur les calculs de distances intertextuelles, voir BARTHELEMY et alii, 2003 ; BRUNET, 2003.

10. J'ai retiré les trois Vies de Pélagie et de Vitus, qui pesaient lourd dans le calcul.

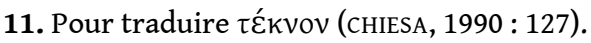

12. Ici, les Passions de Pélagie et de Vitus n'ont pas été retirées, car, dans ce calcul, elles ne pèsent pas aussi lourd que dans celui de la distance lexicale.

13. Dans ce calcul, les vocables sont regroupés d'après leur catégorie : tous les substantifs sont regroupés et considérés comme équivalents, sans distinction de forme ni de sens, et il en va de même pour les autres catégories. Les interjections sont regroupées avec les adverbes, parce qu'elles ont des valeurs faibles.

14. L'indice de corrélation évalue le parallélisme de distribution des deux éléments étudiés. Si les deux éléments sont répartis de façon similaire dans les différents textes du corpus, la corrélation est positive. Si, au contraire, l'un est employé en excédent là où l'autre est employé en déficit, alors elle est négative. Cet indice varie entre +1 et -1 et il devient significatif à partir de 器0,26. 
15. Les conjonctions ne suffisent pas à elles seules pour juger de la complexité d'un texte ; il faut aussi prendre en considération les Ablatifs absolus, les propositions infinitives, relatives et interrogatives qui ne sont pas introduites par des conjonctions. Elles peuvent toutefois servir d'indicateurs, en illustrant, à travers les surplus et les déficits, la tendance de l'auteur à juxtaposer ses phrases, à les coordonner ou à les construire dans des liens de dépendance.

\section{RÉSUMÉS}

$\mathrm{Au}$ fil des siècles, le corpus latin des vitae sanctorum et des passiones martyrum s'est enrichi par l'introduction de nouveaux textes, par la réécriture et l'adaptation d'anciens remis au goût du jour, ainsi que par la traduction d'oeuvres issues du monde byzantin. Le phénomène de la réécriture hagiographique est bien connu et a fait l'objet de nombreux travaux. Celui de la traduction dans ce domaine reste en revanche peu exploité, tant il existe d'obstacles à son étude (absence de répertoire, manque d'éditions critiques, difficultés à identifier les traductions et les modèles grecs, etc.). Le présent travail propose une approche originale du phénomène, basée sur l'utilisation de la statistique linguistique. Cette approche consiste à étudier le latin utilisé par les traducteurs hagiographes pour rendre leur modèle grec et les techniques qu'ils ont adoptées pour le traduire en considérant les traductions non pas une à une, mais d'une manière comparative au moyen de l'analyse quantitative, dans le but de caractériser et de classer les traductions sur base de critères linguistiques, d'identifier et de qualifier des pratiques, des traditions localisées, des spécificités d'école ou de traducteur. La pratique de la traduction est examinée à travers un corpus de 35 textes représentatif des différentes phases de l'histoire de la traduction hagiographique latine au Moyen Âge et des différents foyers de traduction en Italie. Les analyses sont menées sur divers paramètres lexicaux et syntaxiques, en comparant d'abord le corpus, pris dans son ensemble, à un corpus de textes non traduits, puis en comparant entre eux les différents sous-corpus de traductions. Le premier type de comparaison (externe) a pour objectif de mettre en évidence les caractéristiques des traductions en général, le second (interne) de dégager les spécificités de chaque groupe de traductions.

Throughout the Middle Ages, the Latin corpus of vitae sanctorum and passiones martyrum was enriched by introduction of new texts, by rewriting and rehandling of old texts according to the public's tastes, but also by translation of works from the Byzantine world. The phenomenon of hagiographic rewriting was the source of many works and is well known today. The practice of translation in this field however remains little investigated, so many are the obstacles to its study (lack of catalogue and critical editions, difficulties to distinguish between translations and Greek models, etc). This paper proposes an original approach of the phenomenon, based on the use of linguistic statistics. This approach is to study the Latin language used by the hagiographic translators to render their Greek models and the techniques that were implemented to achieve those translations, by considering them not individually but in a comparative way by means of quantitative analysis, for the purpose of the characterization and the classification of the translated works. The practice of translation is considered through a corpus of 35 texts representative of the different stages in the history of Latin hagiographic translation during the Middle Ages and of the various centres of translation in Italy. The analyses are carried out on various lexical and syntactic parameters, first by comparing the corpus, taken as a whole, with a corpus of not translated texts, then by comparing the various sub-corpora of translations 
between them. The aim of the first type of comparison (external) is to highlight the general characteristics of the translations, the aim of the second (intern) is to find the specificities of each group of translations.

\section{INDEX}

Keywords : Translation, Hagiography, Middle Ages, Quantitative Linguistics, Intertextual Distance

Mots-clés : Traduction, hagiographie, Moyen Âge, linguistique quantitative, distance intertextuelle

\section{AUTEUR}

\section{CAROLINE PHILIPPART}

Philologue classique (Université de Liège), docteur en Sciences du Langage (Université de Nice Sophia-Antipolis), responsable de la base médiolatine du Laboratoire d'Analyse Statistique des Langues Anciennes (LASLA) de l'Université de Liège. Après un premier contact avec l'hagiographie médiévale et la statistique au sein du projet Mediolatinitas de l'Université de Namur, elle a soutenu un doctorat sur le thème de « Hagiographie et statistique linguistique : étude d'un corpus de traductions médiolatines d'origine grecque ", pour lequel elle a développé la base de textes hagiographiques lemmatisés du LASLA. Elle poursuit désormais ses recherches dans le domaine de l'hagiologie, de l'analyse textométrique et des questions de traduction du grec au latin. Dans ce cadre, elle a développé des collaborations, en particulier avec Monique Goullet, tout en continuant à enrichir la base par l'introduction de nouveaux textes. En 2013, elle a organisé les premières journées d'étude "Recherches hagiologiques et bases de données". 